\title{
SYNCHRONIZATION AND BASINS OF SYNCHRONIZED STATES IN TWO-DIMENSIONAL PIECEWISE MAPS VIA COUPLING THREE PIECES OF ONE-DIMENSIONAL MAPS
}

\author{
DANIELE FOURNIER-PRUNARET \\ LAAS-CNRS, INSA, University of Toulouse, \\ 7 avenue du Colonel Roche, 31077 Toulouse, France \\ daniele.fournier@laas.fr \\ J. LEONEL ROCHA \\ Instituto Superior de Engenharia de Lisboa, \\ $A D M$ and CEAUL, Rua Conselheiro Emdio Navarro 1, \\ 1959-007 Lisboa, Portugal \\ jrocha@deq.isel.ipl.pt \\ ACILINA CANECO \\ Instituto Superior de Engenharia de Lisboa, \\ $A D M$ and CIMA-UE, Rua Conselheiro Emdio Navarro 1, \\ 1959-007 Lisboa, Portugal \\ acilina@deetc.isel.ipl.pt \\ SARA FERNANDES* and CLARA GRACIO ${ }^{\dagger}$ \\ DMat, CIMA-UE, Universidade de Évora, \\ Largo dos Colegiais 2, 7000 Evora, Portugal \\ *saf@uevora.pt \\ ${ }^{\dagger}$ mgracio@uevora.pt
}

Received November 7, 2012; Revised March 26, 2013

This paper is devoted to the synchronization of a dynamical system defined by two different coupling versions of two identical piecewise linear bimodal maps. We consider both local and global studies, using different tools as natural transversal Lyapunov exponent, Lyapunov functions, eigenvalues and eigenvectors and numerical simulations. We obtain theoretical results for the existence of synchronization on coupling parameter range. We characterize the synchronization manifold as an attractor and measure the synchronization speed. In one coupling version, we give a necessary and sufficient condition for the synchronization. We study the basins of synchronization and show that, depending upon the type of coupling, they can have very different shapes and are not necessarily constituted by the whole phase space; in some cases, they can be riddled.

Keywords: Almost global synchronization; Lyapunov exponents; basins; Lyapunov functions. 


\section{Introduction}

An important feature of our world is the tendency of different systems to achieve common rhythms, namely, the tendency for synchronization. Essentially, the synchronization can be understood as the ability of systems of various types to form a common scheme or to have interaction strength, see for example [Pikovsky et al., 2001; Osipov et al., 2007]. The phenomenon of synchronization occurs when at least two elements are coupled but can also be found in assemblies involving hundreds, thousands or millions of dynamic entities. For instance, we can cite: the insulin-secreting cells in the pancreas [Sherman et al., 1988], the ratchet system [Alatriste \& Mateos, 2006], the behavior of diffusively coupled droplets separated by oil gaps, where each drop contains the reactants of the oscillatory BelousovZhabotinsky reaction [Toiya et al., 2010] or the classical and earliest known (in 1657 by Christian Huygens), the pendulum clock [Caneco et al., 2009; Luo \& Min, 2011]. However, this phenomenon has become even more important and powerful from the moment it was found that synchronization can exist even in systems with chaotic dynamics. This was revealed in the early 90s, with the pioneering studies of Yamada and Fujisaka [1984] and Pecora and Carroll [1990]. Thus, the phenomenon of synchronization has become one of the most active fields of research in nonlinear dynamics. Over the years, different types of synchronization have been characterized, such as the complete synchronization, lag synchronization, generalized synchronization, phase and imperfect phase synchronization [Srinivasan et al., 2012; Wu et al., 2012; Suresh et al., 2012]. In this paper, we also consider the almost global synchronization [Canale \& Monzón, 2008].

Our work is based on previous studies by Hasler and Maistrenko [1987] who have studied in their article various phenomena linked to the synchronization of chaotic systems. They propose two types of coupling that allow synchronization of two onedimensional systems by introducing models of twodimensional piecewise linear transformations. But while they have coupled unimodal maps, we propose to extend their work with bimodal maps. We give a necessary condition of existence of complete synchronization in a plane of coupling parameters. Another purpose is to have a necessary and sufficient condition of complete synchronization and complementary results leading to synchronization.
For this, we propose a complementary study and a discussion in order to obtain global results and not only local. First, let us recall that complete synchronization can be defined as the equality of the state variables while evolving in time, in the case of coupled identical systems. We also consider the notion of almost global synchronization [Canale \& Monzón, 2008], which is defined as the case in which almost all initial conditions (except at most a set of measure zero) in the state space give rise to iterated sequences converging towards the set where the variables of the state space are equal, the diagonal set. For this aim, we consider Lyapunov functions and we study more precisely the eigenvalues and associated eigenvectors on a direction transverse to the diagonal.

We do not introduce new tools or new techniques in our paper. First, we wish to extend the classical tools permitting the study of synchronization in the case of the coupling of bimodal maps, in order to obtain when a necessary and sufficient condition on parameters is for possible synchronization. Then we wish to complete classical works by comparing results obtained from the use of Lyapunov exponents and Lyapunov functions, a discussion using eigenvectors in the phase plane and simulations of basins of attraction. To our knowledge, such work has not yet been done at the same time for the same system. Our aim is to consider the different ways of studying synchronization of chaos or of other attractors in a system, which permit to look at different sides of it. The couplings that we consider are classical ones, but they have not been studied in the case of bimodal maps. We think that our study can permit to extend the work to n-modal maps and also to higher dimensional problems. Moreover, a good knowledge of the synchronization in parameter plane can permit system control in several kinds of applications [Caneco et al., 2011; Wieland et al., 2011].

This article is organized as follows. In Sec. 2, we consider a dynamical system defined by two different coupling versions of two identical piecewise linear bimodal maps and we give a necessary condition to obtain complete synchronization for the two versions, using the transversal Lyapunov exponent. Moreover, we characterize the synchronization manifold as an attractor and measure the synchronization speed. In Sec. 3, our purpose is to obtain a necessary and sufficient condition for the synchronization behavior and to know which initial 
conditions in the phase plane give rise to synchronization. In Sec. 4, we present some properties of the basins of synchronized states for two coupling versions, namely, basins can be riddled. Our basins are obtained by numerical simulations, coupled with geometrical properties of critical lines. Finally, in the last section, we summarize the results and open routes for future work.

\section{Synchronization of Chaotic Piecewise Linear Bimodal Maps}

One of the possible criteria for synchronization is that all transversal Lyapunov exponents of trajectories are negative. Nevertheless, this condition is not sufficient. In [Pecora \& Carroll, 1990, 1991] it was established that synchronization can be achieved provided that all the transversal Lyapunov exponents are negative. Since then, some authors [Shuai et al., 1997] have reported their computational experiments showing that apparently, it is possible to achieve synchronization without the negativity of all transversal Lyapunov exponents and some others, see [Cao \& Lu, 2006] and references therein, have reported that sometimes there is a brief lack of synchronization in the regions where all the transversal Lyapunov exponents are negative. How to explain these situations?

In fact, there is a numerical trap when coupling identical systems. Near the synchronization manifold, the two identical systems look like in complete synchronization due to finite precision of numerical calculations [Pikovsky et al., 2001]. As a matter of fact, the negativity of the transversal Lyapunov exponents is a necessary condition for the stability of the synchronized state [Boccaletti et al., 2002]. Indeed, the conditional or transversal Lyapunov exponent is related to the logarithm average of the distance of the solutions on the transversal manifold to the solutions on the synchronization manifold. The negativity of the transversal Lyapunov exponent is a mathematical expression of the decreasing of the logarithm average. So, if there is a strong convergence of this distance to zero, this average must decrease to zero. But the converse is not true. Indeed, even when all the transversal Lyapunov exponents are negative, it is possible that some orbits escape from the synchronization manifold. This is only a weak synchronization, in the sense of Milnor [Pikovsky et al., 2001]. Only Lyapunov functions give necessary and sufficient conditions for the stability of the synchronization manifold. Besides the fact that the stability based on the negativity of the transversal Lyapunov exponents be only a weak stability, there are other phenomena like bubbling, riddling and blowout bifurcation that can explain the brief and persistent events of desynchronization in the region where all the transversal Lyapunov exponents are negative, see [Boccaletti et al., 2002] and references therein.

In this section we consider a dynamical system defined by two different coupling versions of two identical piecewise linear bimodal maps. We are concerned with the range parameter for which there is synchronization of these maps, using the transversal Lyapunov exponent. Moreover, we characterize the synchronization manifold as an attractor and measure the synchronization speed.

\subsection{Coupling of two identical piecewise linear bimodal maps}

Let $f:[0,1] \rightarrow[0,1]$ be a bimodal map, defined by

$$
\begin{aligned}
f(x)= & |1-| 3 x-1|| \\
= & \begin{cases}3 x, & 0 \leq x<\frac{1}{3} \\
-3 x+2, & \frac{1}{3} \leq x<\frac{2}{3} \\
3 x-2, & \frac{2}{3} \leq x<1 .\end{cases}
\end{aligned}
$$

Taking into account the one-dimensional map (1), we consider two kinds of coupling [Hasler \& Maistrenko, 1987] to study synchronization phenomena. Version 1 of the coupling is given by $\left(x_{k+1}, y_{k+1}\right)=F\left(x_{k}, y_{k}\right)$, with $F$ defined by

$$
\left\{\begin{array}{l}
x_{k+1}=f\left(x_{k}\right)+\delta\left(y_{k}-x_{k}\right) \\
y_{k+1}=f\left(y_{k}\right)+\varepsilon\left(x_{k}-y_{k}\right) .
\end{array}\right.
$$

Version 2 of the coupling is given by $\left(x_{k+1}, y_{k+1}\right)=$ $G\left(x_{k}, y_{k}\right)$, with $G$ defined by

$$
\left\{\begin{array}{l}
x_{k+1}=f\left[x_{k}+\delta\left(y_{k}-x_{k}\right)\right] \\
y_{k+1}=f\left[y_{k}+\varepsilon\left(x_{k}-y_{k}\right)\right] .
\end{array}\right.
$$

In both cases, $k \in \mathbb{N}_{0}, x_{k}$ and $y_{k}$ are the state variables, $\delta$ and $\varepsilon$ are real parameters. Both versions of coupling are bidirectional, since the systems $x_{k+1}=f\left(x_{k}\right)$ and $y_{k+1}=f\left(y_{k}\right)$ are connected in such a way that they mutually influence each other's behavior. In version 1 , the map $F$ can generally be 


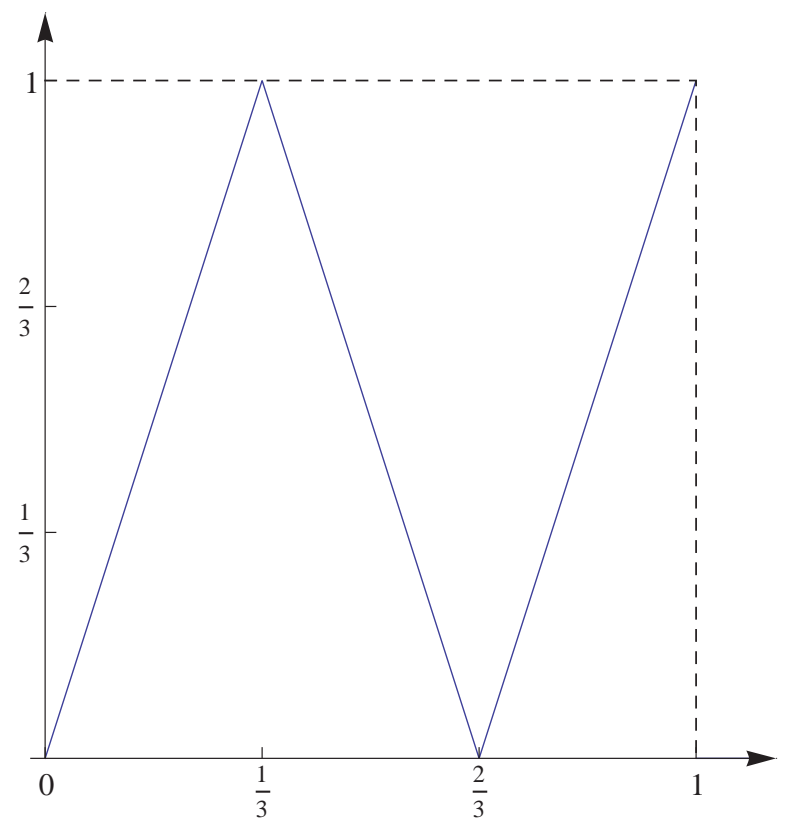

Fig. 1. The map (1).

defined from $\mathbb{R}^{2}$ to $\mathbb{R}^{2}$, but we mainly consider the situation in $[0,1]^{2}$, when there exists bounded iterated sequences. The two coupling parameters $\delta$ and $\varepsilon$ are positive. In version 2 , the map $G$ is defined from $[0,1]^{2}$ to $[0,1]^{2}$ when $(\delta, \varepsilon) \in[0,1]^{2}$.

Considering (1), we can write the map $F$ of $(2)$, coupling version 1 , as

$$
\left\{\begin{array}{l}
x_{k+1}=|1-| 3 x_{k}-1||+\delta\left(y_{k}-x_{k}\right) \\
y_{k+1}=|1-| 3 y_{k}-1||+\varepsilon\left(x_{k}-y_{k}\right)
\end{array}\right.
$$

and the map $G$ of (3), coupling version 2 , as

$$
\left\{\begin{array}{l}
x_{k+1}=|1-| 3\left[x_{k}+\delta\left(y_{k}-x_{k}\right)\right]-1|| \\
y_{k+1}=|1-| 3\left[y_{k}+\varepsilon\left(x_{k}-y_{k}\right)\right]-1||
\end{array}\right.
$$

or, equivalently

$$
\left\{\begin{array}{l}
x_{k+1}=|1-| \alpha x_{k}+(3-\alpha) y_{k}-1|| \\
y_{k+1}=|1-| \beta y_{k}+(3-\beta) x_{k}-1||
\end{array}\right.
$$

where $\alpha=3-3 \delta$ and $\beta=3-3 \varepsilon$. This version of coupling corresponds to the family of maps considered in [Manjunath \& Fournier-Prunaret, 2011], defined on $[0,1]^{2}$, by

$$
\begin{aligned}
G_{\alpha, \beta}(x, y)= & (|1-| \alpha x+(3-\alpha) y-1||, \\
& |1-|(3-\beta) x+\beta y-1||)
\end{aligned}
$$

with the parameters $(\alpha, \beta)$ taking values in $[0,3]^{2}$ to have the square $[0,1]^{2}$ mapped into itself $\left((\alpha, \beta) \in[0,3]^{2}\right.$ is equivalent to $\left.(\delta, \varepsilon) \in[0,1]^{2}\right)$. Some important properties of the map $G_{\alpha, \beta}$ have been previously studied in [Manjunath \& FournierPrunaret, 2011].

\subsection{Synchronization range parameter and transversal Lyapunov exponent}

Systems (2) and (3) are two different ways of coupling the same function $f$. When the coupled systems are identical, synchronization appears as the equality of the state variables while evolving in time. This type of synchronization is referred to in the literature as complete or identical synchronization.

The maps $F$ and $G$ defined by (4) and (5), respectively, keep invariant the segment of the diagonal set

$$
S=\left\{(x, y) \in \mathbb{R}^{2}: 0 \leq x=y \leq 1\right\} .
$$

The straight line $x=y$ is the subspace where the two dynamical systems $x_{k+1}=f\left(x_{k}\right)$ and $y_{k+1}=$ $f\left(y_{k}\right)$ are synchronized.

Definition. The synchronization manifold $S$ is said to be globally asymptotically stable (or the dynamical systems defined by (2) or (3) are globally asymptotically synchronized) if for any $\eta>0$, there exists $k_{0}$, such that for all $k>k_{0}$,

$$
\left\|x_{k}-y_{k}\right\| \leq \eta \text {. }
$$

A trajectory of the dynamical system defined by (2) or (3) synchronizes if it is bounded and $\lim _{k \rightarrow \infty}\left|x_{k}-y_{k}\right|=0$. The synchronization stability study of identically coupled systems can be formulated by addressing the question of the stability of the synchronization manifold $(x \equiv y)$, or equivalently, by studying the temporal evolution of the synchronization error $e_{k} \equiv x_{k}-y_{k}$.

In order to check the synchronization we have to investigate if a trajectory that starts near $S$ converges or not to a trajectory on $S$. The speed of mutual convergence or divergence of these trajectories is measured by the Lyapunov exponents.

\subsubsection{Lyapunov exponents}

One of the most important properties of a chaotic system is the sensitivity to initial conditions. A way to measure the sensitivity with respect to initial 
conditions is to compute the average rate at which nearby trajectories diverge from each other. The computation of the Lyapunov exponents gives the average rate of convergence or divergence of two trajectories from each other.

The Lyapunov exponents of a trajectory $x_{k}$ is defined by

$$
\lambda=\lim _{k \rightarrow+\infty} \frac{1}{k} \sum_{j=0}^{k-1} \ln \left|f^{\prime}\left(x_{j}\right)\right|
$$

whenever it exists [Hasler \& Maistrenko, 1987]. Note that, if $x_{j}=\frac{1}{3}$ or $x_{j}=\frac{2}{3}$, for some $j$, then, $f^{\prime}\left(x_{j}\right)$ does not exist. The number of such trajectories $x_{j}$ is countably infinite, so the Lebesgue measure of these initial conditions is zero. The question of existence of the limit in (7) and its independence of a certain particular trajectory is associated with a unique probability density $\rho(x)$, that is $f$-invariant. The $f$-invariant probability density $\rho(x)$ on $[0,1]$ for the piecewise linear map (1) verifies

$$
\rho(x)=\frac{1}{3}\left[\rho\left(\frac{x}{3}\right)+\rho\left(\frac{x-2}{-3}\right)+\rho\left(\frac{x+2}{3}\right)\right] .
$$

It can be shown that the only probability density which satisfies (8) is the constant probability on $[0,1], \rho(x)=1$. From Birkhoff's ergodic theorem, it follows that the asymptotic distribution of the points of almost all trajectories is uniform. For almost all initial conditions, the Lyapunov exponent defined by (7) exists, is designated by natural Lyapunov exponent and may be expressed as

$$
\lambda^{\text {nat }}=\int_{0}^{1} \ln \left|f^{\prime}(x)\right| \rho(x) d x
$$

For the piecewise linear map (1), we have

$$
\lambda=\lambda^{\text {nat }}=\ln (3) .
$$

\subsubsection{Synchronization domain}

The map $F$ of coupling version 1, defined by system (4), has nine determinations, depending on the sign of the terms inside the brackets $|\cdot|$ in the expression of the map, i.e. depending on the region $R_{i j}$ where $(x, y)$ belongs, see Fig. 2 . We consider $R_{i j}$ as the open regions, which are limited by the straight lines $l_{i}, i=1,2,3,4$, where $l_{1} \equiv y=1 / 3$, $l_{2} \equiv x=1 / 3, l_{3} \equiv y=2 / 3$ and $l_{4} \equiv x=2 / 3$. These line segments are the closure of a subset on which

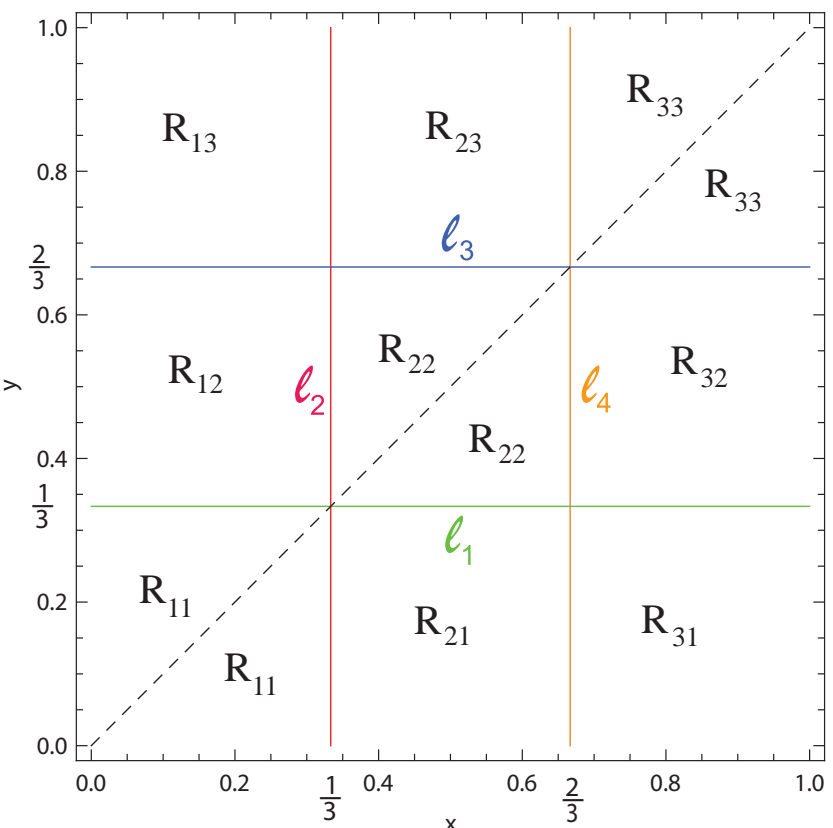

Fig. 2. The regions $R_{i j}$, limited by the lines $l_{1} \equiv y=1 / 3$, $l_{2} \equiv x=1 / 3, l_{3} \equiv y=2 / 3$ and $l_{4} \equiv x=2 / 3$, constitute a partition of the square $[0,1]^{2}$. The expression of the map $F$ of coupling version 1 depends on the region $R_{i j}$.

$F$ fails to be differentiable, and we call these line segments critical lines [Mira et al., 1996].

In region $R_{11}$, the version 1 of the coupling is given by

$$
\left\{\begin{array}{l}
x_{k+1}=3 x_{k}+\delta\left(y_{k}-x_{k}\right) \\
y_{k+1}=3 y_{k}+\varepsilon\left(x_{k}-y_{k}\right) .
\end{array}\right.
$$

Respectively, in $R_{22}(11)$ and $R_{33}(12)$, one has

$$
\begin{aligned}
& \left\{\begin{array}{l}
x_{k+1}=-3 x_{k}+2+\delta\left(y_{k}-x_{k}\right) \\
y_{k+1}=-3 y_{k}+2+\varepsilon\left(x_{k}-y_{k}\right)
\end{array}\right. \\
& \left\{\begin{array}{l}
x_{k+1}=3 x_{k}-2+\delta\left(y_{k}-x_{k}\right) \\
y_{k+1}=3 y_{k}-2+\varepsilon\left(x_{k}-y_{k}\right) .
\end{array}\right.
\end{aligned}
$$

In regions $R_{i j}, i, j=1,2,3, i \neq j$, the version 1 of the coupling is given by combinations of expressions of $x_{k+1}$ and $y_{k+1}$ obtained from (10), (11) or (12), depending where the point $\left(x_{k}, y_{k}\right)$ is located regarding the straight lines $l_{i}, i=1,2,3,4$.

For points on the synchronization subspace $S$, only the regions $R_{i i}$ matter, the Jacobian matrix of the system (4) for these points, where $x=y$, is

$$
D F\left[\begin{array}{l}
x \\
x
\end{array}\right]=\left[\begin{array}{cc}
c-\delta & \delta \\
\varepsilon & c-\varepsilon
\end{array}\right]
$$


where

$$
c=c(x)= \begin{cases}3 & \text { if } x \in\left[0, \frac{1}{3}[\cup] \frac{2}{3}, 1\right] \\ -3 & \text { if } x \in] \frac{1}{3}, \frac{2}{3}[.\end{cases}
$$

The eigenvalues of the Jacobian $D F$ are

$$
\mu_{1}(x)=c \text { and } \mu_{2}(x)=c-\varepsilon-\delta .
$$

The corresponding eigenvectors are

$$
v_{1}=(1,1) \quad \text { and } \quad v_{2}=(\delta,-\varepsilon) .
$$

For the map $G$ of coupling version 2, defined by system (5), there are nine determinations, depending on the sign of the terms inside the brackets $|\cdot|$ in (5), i.e. depending on the region $R_{i j}$ where $(x, y)$ belongs, see Fig. 3 .

The regions $R_{i j}$ are limited by the straight lines contained in $I^{2}$ given by $\alpha x+(3-\alpha) y-1=0$, $\alpha x+(3-\alpha) y-1=1,(3-\beta) x+\beta y-1=0$ and $(3-\beta) x+\beta y-1=1$, which are respectively denoted by $l_{1}, l_{2}, l_{3}$ and $l_{4}$. As in the case of the map $F$, these line segments are the closure of a subset on which $G_{\alpha, \beta}$ fails to be differentiable, and are called

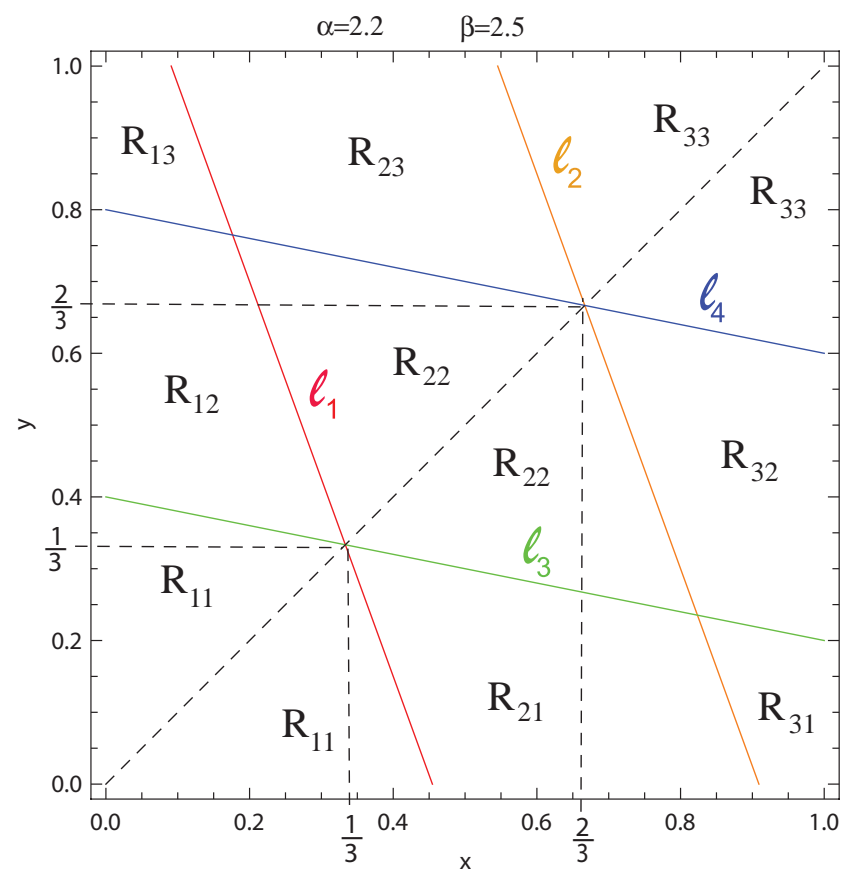

Fig. 3. As for the map $F$, the expression of the map $G$ of coupling version 2 depends on the region $R_{i j}$, which are limited by the lines $l_{1}, l_{2}, l_{3}$ and $l_{4}$. The regions $R_{i j}$ constitute a partition of the square $[0,1]^{2}$. The lines $l_{i}, i=1,2,3,4$ are not horizontal nor vertical, but the situation is very similar to that of the map $F$. critical lines [Mira et al., 1996]. It is straightforward to observe that the critical line $l_{1}$ is parallel to $l_{2}$, and $l_{3}$ is parallel to $l_{4}$ regardless of $\alpha, \beta$. The slopes of $l_{1}$ and $l_{2}$ are equal to $\alpha /(\alpha-3)$ and the slopes of $l_{3}$ and $l_{4}$ are equal to $(\beta-3) / \beta$. They are always negatives. The slopes of $l_{3}$ and $l_{4}$ are less than the slopes of $l_{1}$ and $l_{2}$ iff $\alpha+\beta<3$. When $\alpha+\beta<3$, the slopes of $l_{3}$ and $l_{4}$ are less than the slopes of $l_{1}$ and $l_{2}$. When $\alpha+\beta>3$, the slopes of $l_{3}$ and $l_{4}$ are greater than the slopes of $l_{1}$ and $l_{2}$ and regions $R_{i j}$ are inverted regarding the diagonal $x=y$. When $\alpha+\beta$ belongs to [2,4], regions $R_{13}$ and $R_{31}$ are outside of the square. These lines define the boundaries of regions $R_{i j}, i, j=1,2,3$, where $G_{\alpha, \beta}$ is defined by one of the nine determinations.

In $R_{11}$, version 2 of the coupling is given by

$$
\left\{\begin{array}{l}
x_{k+1}=3\left[x_{k}+\delta\left(y_{k}-x_{k}\right)\right] \\
y_{k+1}=3\left[y_{k}+\varepsilon\left(x_{k}-y_{k}\right)\right] .
\end{array}\right.
$$

Respectively, in $R_{22}(17)$ and $R_{33}$ (18), one has

$$
\begin{aligned}
& \left\{\begin{array}{l}
x_{k+1}=-3\left[x_{k}+\delta\left(y_{k}-x_{k}\right)\right]+2 \\
y_{k+1}=-3\left[y_{k}+\varepsilon\left(x_{k}-y_{k}\right)\right]+2
\end{array}\right. \\
& \left\{\begin{array}{l}
x_{k+1}=3\left[x_{k}+\delta\left(y_{k}-x_{k}\right)\right]-2 \\
y_{k+1}=3\left[y_{k}+\varepsilon\left(x_{k}-y_{k}\right)\right]-2 .
\end{array}\right.
\end{aligned}
$$

As in the case of the map $F$, in regions $R_{i j}$, $i, j=1,2,3, i \neq j$, version 2 of the coupling is given by combinations of expressions of $x_{k+1}$ and $y_{k+1}$ obtained from (16), (17) or (18), depending where the point $\left(x_{k}, y_{k}\right)$ is located on the straight lines $l_{i}, i=1,2,3,4$.

For the study of the Jacobian matrix of system (5) for points $(x, y) \in S$, only the regions $R_{i i}$ matter, so, for these points the Jacobian is

$$
D G\left[\begin{array}{l}
x \\
x
\end{array}\right]=c\left[\begin{array}{cc}
1-\delta & \delta \\
\varepsilon & 1-\varepsilon
\end{array}\right]=c\left[\begin{array}{cc}
\frac{\alpha}{3} & \frac{3-\alpha}{3} \\
\frac{3-\beta}{3} & \frac{\beta}{3}
\end{array}\right]
$$

where $c$ is defined in (13). In this case, the eigenvalues of $D G$ are

$$
\mu_{1}(x)=c \quad \text { and } \quad \mu_{2}(x)=c(1-\delta-\varepsilon)
$$

and the corresponding eigenvectors are

$$
\begin{aligned}
& v_{1}=(1,1) \text { and } \\
& v_{2}=(\delta,-\varepsilon)=\left(\frac{3-\alpha}{3},-\frac{3-\beta}{3}\right) .
\end{aligned}
$$


For both cases, (4) version 1 or (5) version 2, the eigenvectors do not depend on $x$, they are also eigenvectors of

$$
D_{k}=\frac{\partial G}{\partial x}\left(x_{k-1}\right) \frac{\partial G}{\partial x}\left(x_{k-2}\right) \cdots \frac{\partial G}{\partial x}\left(x_{0}\right) .
$$

The two eigenvalues of $D_{k}$ are

$$
\mu_{i}=\mu_{i}\left(x_{k-1}\right) \mu_{i}\left(x_{k-2}\right) \cdots \mu_{i}\left(x_{0}\right)
$$

with $i=1,2$. The corresponding Lyapunov exponents are

$$
\lambda_{i}=\lim _{k \rightarrow+\infty} \frac{1}{k} \sum_{j(0)}^{k-1} \ln \left|\mu_{i}\left(x_{j}\right)\right| .
$$

Note that, for both cases, (4) version 1 and (5) version 2 , the first eigenvector belongs to the subspace $S$. Thus, the first Lyapunov exponent, which will be denoted by $\lambda_{\|}$, corresponds to the motion in the subspace $S$ and this motion is given by the one-dimensional dynamical system $x_{k}=f^{k}\left(x_{0}\right)$. In particular, we obtain

$$
\lambda_{1}=\lambda_{\|}=\ln (3) .
$$

The second eigenvector is transversal to $S$ and the corresponding Lyapunov exponent is called transversal Lyapunov exponent, which will be denoted by $\lambda_{\perp}$. Note that, for the case (4) version 1 , the eigenvalue $\mu_{2}$ depends on $x$. In the case (5) version 2 , the eigenvalue $\mu_{2}$ also depends on $x$, however the corresponding Lyapunov exponent does not and we obtain

$$
\lambda_{2}=\lambda_{\perp}=\ln |c(1-\delta-\varepsilon)|=\ln |\alpha+\beta-3| .
$$

Using the one-dimensional invariant probability density $\rho(x)=1$ on $S$, the value of $\lambda_{i}$, for almost all trajectories in $S$, can be computed by applying Birkhoff's ergodic theorem, i.e.

$$
\lambda_{i}^{\text {nat }}=\int_{0}^{1} \ln \left|\mu_{i}(x)\right| \rho(x) d x .
$$

For both cases, the natural parallel Lyapunov exponent verifies

$$
\lambda_{\|}^{\text {nat }}=\lambda_{1}=\lambda_{\|}=\ln (3) .
$$

In the case (4) version 1 , we obtain the natural transversal Lyapunov exponent

$$
\lambda_{\perp}^{\text {nat }}=\lambda_{2}=\frac{1}{3} \ln \left(|3-\varepsilon-\delta|^{2}|-3-\varepsilon-\delta|\right) .
$$

In the case (5) version 2, the natural transversal Lyapunov exponent is

$$
\lambda_{\perp}^{\text {nat }}=\lambda_{2}=\lambda_{\perp}=\ln |\alpha+\beta-3| .
$$

If $\lambda_{\perp}^{\text {nat }}$ is negative, the trajectories beginning close to $S$ are in the mean attracted to $S$ and the system synchronizes. In the case (4) version $1, \lambda_{\perp}^{\text {nat }}$ is negative if $(3-d)^{2}|3+d|<1$, where $d=\varepsilon+\delta$, i.e. $d_{1}<\varepsilon+\delta<d_{2}$, where $d_{1}=2.57653 \ldots$ and $d_{2}=3.39543 \ldots$ are real positive solutions of $(3-d)^{2}|3+d|=1$. See Fig. 4. According to [Hasler \& Maistrenko, 1987], under these conditions, the subspace $S$ is an attractor with a locally riddled basin. In the case (5) version $2, \lambda_{\perp}^{\text {nat }}$ is negative if $|\alpha+\beta-3|<1$. Note that, the synchronization regions obtained theoretically are similar to the ones obtained by numerical simulations. See Figs. 5 and 6 .

So, we proved the existence of a synchronization range parameter to each case of coupling. The next result follows.

Property 1. Consider the coupling of two identical piecewise linear bimodal maps (1).

(1) If the coupling is defined by (4), with two different coupling positive parameters $(\varepsilon, \delta)$, then the natural transversal Lyapunov exponent is

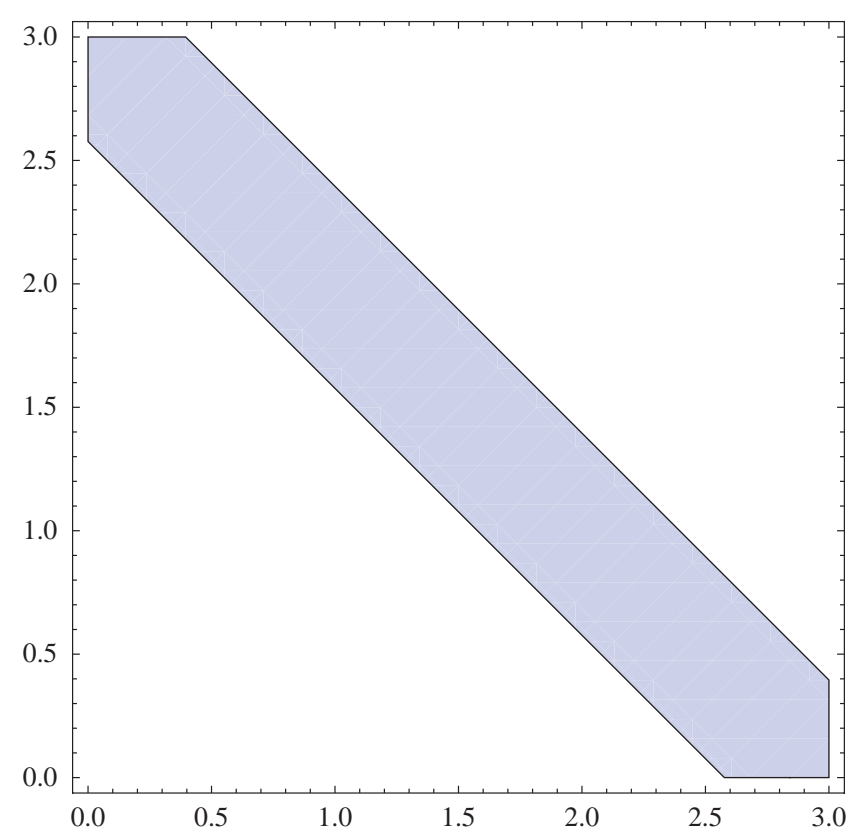

Fig. 4. Parameter region $2.57653 \ldots<\varepsilon+\delta<3.39543 \ldots$ where synchronization occurs for the coupling (4) version 1 , of two identical piecewise linear bimodal maps (1). 
negative if $d_{1}<\varepsilon+\delta<d_{2}$, where $d_{1}$ and $d_{2}$ are real positive solutions of $(3-d)^{2}|3+d|=1$.

(2) If the coupling is defined by (5), with two different coupling parameters $(\alpha, \beta)$, each one in $[0,3]$, then the natural transversal Lyapunov exponent is negative if $2<\alpha+\beta<4$.

So, by this property, the fact that the natural transversal Lyapunov exponent is negative means that almost all synchronized trajectories, i.e. almost all trajectories near $S$ are transversally attracting, which implies that $S$ is an attractor in the weak sense of Milnor [Alexander et al., 1992; Ashwin et al., 1996]. We have obtained synchronization range parameter, in terms of the coupling parameters, for both types of coupling.

\section{3. $S$ as an attractor and synchronization speed}

Consider the case (4) version 1. Note that, the transversal Lyapunov exponent for the constant trajectories at the fixed point $(0,0)$ is $\lambda_{\perp}=\ln \mid 3-$ $\varepsilon-\delta \mid$, which is negative if $2<\varepsilon+\delta<4$. On the other hand, the transversal Lyapunov exponent for the constant trajectories at the fixed point $(1 / 2,1 / 2)$ is $\lambda_{\perp}=\ln |-3-\varepsilon-\delta|$, which is negative if $-4<\varepsilon+\delta<-2$, but we do not consider this case. Thus, for any positive values of the coupling parameters $(\varepsilon, \delta)$, we have at least one of the two transversal Lyapunov exponents at $(0,0)$ or $(1 / 2,1 / 2)$ that is positive. Furthermore, these fixed points of $F$ have preimages dense in $S$ [Hasler \& Maistrenko, 1987], then, always a dense set exists on $S$ of initial conditions that leads to trajectories with positive transversal Lyapunov exponents.

However, in the case (5) version 2 , the transversal Lyapunov exponent for any trajectory in $S$, is $\lambda_{\perp}^{\text {nat }}=\lambda_{2}=\lambda_{\perp}=\ln |\alpha+\beta-3|$, according to (19) and (23), which is negative if $2<\alpha+\beta<4$. In particular, the value of $\lambda_{\perp}^{\text {nat }}$ is attained for the constant trajectories sitting on the fixed points. So, all synchronized trajectories are transversally attracting. Outside the region $2<\alpha+\beta<4$, the transversal Lyapunov exponent is positive. Once more, the preimages of the fixed points give a dense set on $S$ of initial conditions, that leads to trajectories with positive transversal Lyapunov exponents.

We have proved the following results.

Property 2. Consider the coupling of two identical piecewise linear bimodal maps (1).
(1) If the coupling is defined by (4), with two different coupling positive parameters $(\varepsilon, \delta)$, then the system has trajectories in the synchronization subspace $S$ with a positive transversal Lyapunov exponent, for any value of the positive coupling parameters $(\varepsilon, \delta)$. The initial conditions of these trajectories are dense in $S$.

(2) If the coupling is defined by (5), with two different coupling parameters $(\alpha, \beta)$, then the transversal Lyapunov exponent is negative if $2<\alpha+\beta<4$. For any values of the parameters $(\alpha, \beta)$ in $[0,3]^{2}$ and outside of the closure of the region $2<\alpha+\beta<4$, there is a dense set of initial conditions that generate trajectories with positive transversal Lyapunov exponent.

Property 3. Consider the coupling of two identical piecewise linear bimodal maps (1) defined by (4). If the natural transversal Lyapunov exponent is negative, then $S$ is an attractor with a locally riddled basin.

This result is a consequence of (1) of Properties 1 and 2. By Property 1 there exists a region $d_{1}<\varepsilon+\delta<d_{2}$, where the natural transversal Lyapunov exponent is negative and by Property 2 there exists trajectories in the synchronization subspace $S$, with a positive transversal Lyapunov exponent. So, there is a neighborhood $U$ of $S$ such that in any neighborhood $V$ of any point in $S$, there is a set of points in $V \cap U$ of positive measure which leave $U$ in a finite time. For more details, see [Hasler \& Maistrenko, 1987; Alexander et al., 1992; Ashwin et al., 1996].

Proposition 1. Consider the coupling of two identical piecewise linear bimodal maps, defined by (5).

(1) If $|\alpha+\beta-3|<1$, then the manifold $S$ is a globally asymptotically stable attractor.

(2) If $(\alpha, \beta)$ is outside of the closure of the region $|\alpha+\beta-3|<1$, then the manifold $S$ has a locally riddled basin of attraction.

(3) If $x_{k}$ and $y_{k}$ are trajectories starting from $x_{0}$ and $y_{0}$, respectively, and are always in the same linear region, until time $k$, then these trajectories synchronize with an exponential synchronization speed,

$$
\left|x_{k}-y_{k}\right|=\phi^{k}\left|x_{0}-y_{0}\right|
$$

where $\phi=|\alpha+\beta-3|$.

Proof. In (2) of Property 1, it is established that the natural transversal Lyapunov exponent is 
negative if $|\alpha+\beta-3|<1$. This region coincides with the region where the transversal Lyapunov exponent, of all trajectories in $S$, are negative, by (2) of Property 2. Therefore, the manifold $S$ is a globally asymptotically stable attractor.

On the other hand, considering again, (2) of Properties 1 and 2 , for any values of $(\alpha, \beta)$ outside of the closure of the region $|\alpha+\beta-3|<1$, there is a dense set of initial conditions that generate trajectories with positive transversal Lyapunov exponent. So, the manifold $S$ has a locally riddled basin of attraction [Hasler \& Maistrenko, 1987; Alexander et al., 1992; Ashwin et al., 1996].

Moreover, for trajectories in the same linear region, until the time $k$, the rate of synchronization is proved:

$$
\begin{aligned}
\left|x_{k}-y_{k}\right|= & f\left[x_{k-1}+\delta\left(y_{k-1}-x_{k-1}\right)\right] \\
& -f\left[y_{k-1}+\varepsilon\left(x_{k-1}-y_{k-1}\right)\right] \\
= & 3|1-(\delta+\varepsilon)|\left|x_{k-1}-y_{k-1}\right| \\
= & |\alpha+\beta-3|\left|x_{k-1}-y_{k-1}\right| \\
= & \phi^{k}\left|x_{0}-y_{0}\right|
\end{aligned}
$$

where $\phi=|\alpha+\beta-3|$.

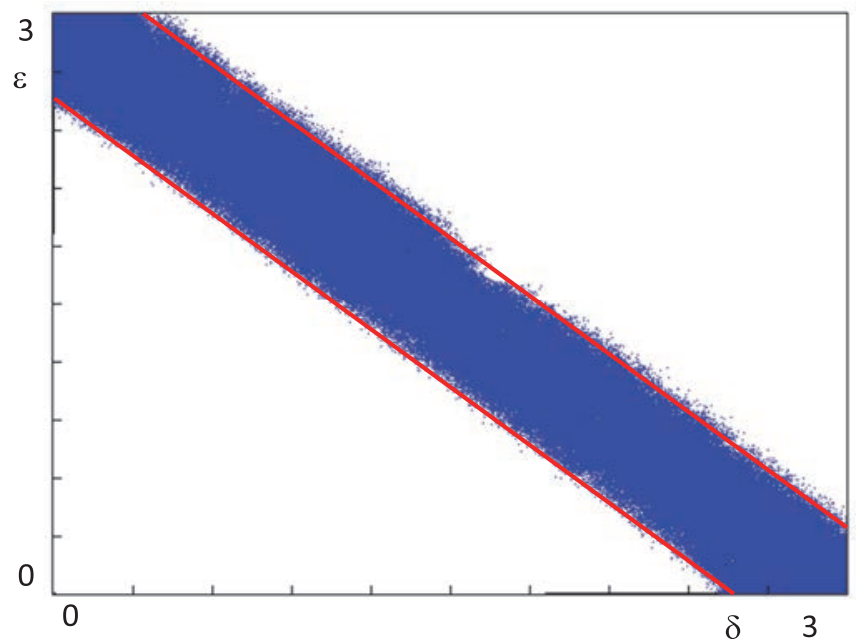

Fig. 5. In blue, region of synchronization in the plane $(\delta, \varepsilon)$ for the map $F(4)$ version 1 . This figure has been obtained by numerical simulations using 10000 iterations for each point. The red straight lines indicate the theoretical limits obtained from Properties 1 and 2. Due to the existence of riddled basins with a fractal boundary for the chaotic attractor on the diagonal (see section on basins), the limits of the synchronization region in the parameter plane that are numerically obtained are not clearly defined.

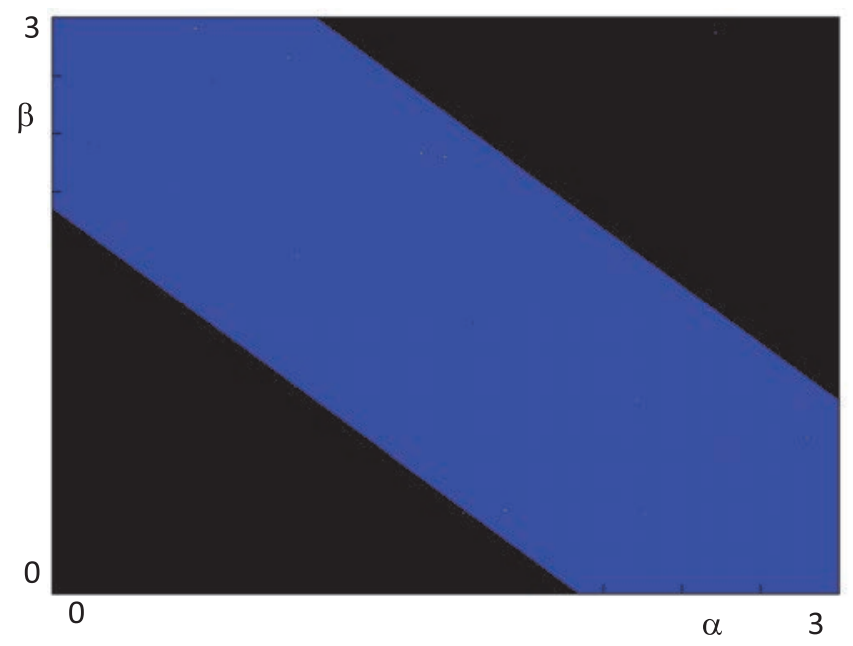

Fig. 6. In blue, region of synchronization in the plane $(\alpha, \beta)$ for map $G$ (5) version (2). The black part corresponds to the existence of a chaotic attractor outside the diagonal. It has been proved in [Manjunath \& Fournier-Prunaret, 2011] that there is a domain in the parameter plane where the map has the property of mixing (inside the black part). This figure has been obtained by numerical simulations using 10000 iterations for each point. In this case, the basin boundaries of the attractor on the diagonal are not fractal, so the limits of the synchronization region in the parameter plane are clearly defined from a numerical point of view and correspond to the theoretical limits obtained in Properties 1 and 2.

The equality (24) characterizing the speed synchronization in version 2 coupling, shows the different qualitative behaviors from version 1 coupling.

Figures 5 and 6 are obtained with numerical simulations in the parameter planes $(\delta, \varepsilon)$ and $(\alpha, \beta)$, respectively, and correspond to the theoretical results obtained using versions 1 and 2 coupling.

Remark 2.1. If $\alpha=\beta$ and $(x, y) \in R_{i i}$, with $i=$ $1,2,3$, we may write the system (5) in the form

$$
\left\{\begin{array}{l}
x_{k+1}=f_{i}\left(x_{k}\right)+\delta\left[f_{i}\left(y_{k}\right)-f_{i}\left(x_{k}\right)\right] \\
y_{k+1}=f_{i}\left(y_{k}\right)+\delta\left[f_{i}\left(x_{k}\right)-f_{i}\left(y_{k}\right)\right],
\end{array}\right.
$$

with $\delta=\varepsilon=(3-\alpha) / 3$ and

$$
f_{i}(x)= \begin{cases}3 x, & \text { if } i=1 \\ -3 x+2, & \text { if } i=2 \\ 3 x-2, & \text { if } i=3 .\end{cases}
$$

This system may be considered a network with only two nodes. The graph underlying this network is complete, so the Laplacian matrix is

$$
L=\left[\begin{array}{rr}
1 & -1 \\
-1 & 1
\end{array}\right] \text {. }
$$


According to $\mathrm{Li}$ and Chen [2003], the bidirectional coupled system (25) is synchronized if the coupling parameter $\delta$ belongs to the synchronization interval, defined by

$$
\frac{1-e^{-\lambda}}{\left|\gamma_{2}\right|}<\delta<\frac{1+e^{-\lambda}}{\left|\gamma_{N}\right|}
$$

where $\lambda$ is the Lyapunov exponent of $f, \gamma_{2}$ is the smaller nonzero eigenvalue of $L$ and $\gamma_{N}$ is the largest eigenvalue of $L$. In this case, $\lambda=\ln \left|f_{i}^{\prime}(x)\right|=\ln (3)$ and the eigenvalues of $L$ are 0 and 2 , so, we have $\gamma_{2}=\gamma_{N}=2$. Then, the synchronization interval is $1 / 3<\delta<2 / 3$, that is, $1<\alpha<2$, which confirms the previous result, $2<\alpha+\beta<4$.

\section{Global Behavior and Basins of the Synchronized States}

In the previous section, we have given a necessary condition to obtain complete synchronization for the two versions of coupling that we have considered. Indeed, the negativity of the transversal Lyapunov exponents is only a necessary condition for the stability of the synchronized state. Moreover, the previous study is a local one and only permits to detect if initial conditions located inside neighborhoods of the diagonal will give rise to trajectories converging toward the diagonal. Of course, it should be of the greatest interest to have a necessary and sufficient condition for the synchronization behavior and to know which initial conditions in the phase plane will give rise to synchronization. In this section, we intend to partially answer these questions. First, we consider Lyapunov functions in order to prove that the conditions that have been obtained in the previous section concerning parameter values are also sufficient conditions. Secondly, we propose to discuss the values of eigenvalues and eigenvectors in regions $R_{i j}$ in order to find the basins of the synchronized states. We obtain results when considering the regions $R_{i i}, i=1,2,3$, but in the regions $R_{i j}, i, j=1,2,3, i \neq j$, the situation is not so easy.

\subsection{Lyapunov functions}

The stability of the synchronization manifold $S$ can also be studied by the use of the Lyapunov function, which gives a necessary and sufficient condition for stability. To the study of temporal evolution of the synchronization error $e \equiv x=y$, the Lyapunov function $V(e)$ is considered.

A continuous scalar function $V: \subseteq \mathbb{R}^{n} \rightarrow \mathbb{R}$ is said to be a Lyapunov function if it is a locally positive-definite function, i.e.

$$
V(0)=0 \quad \text { and } \quad V(x)>0, \quad \forall x \in U \backslash\{0\}
$$

where $U$ is a neighborhood of $x=0$.

To get the conditions for the global stability of synchronization of two trajectories $x_{k}$ and $y_{k}$, we consider the Lyapunov function

$$
V_{k}=\left(x_{k}-y_{k}\right)^{2},
$$

defined as [Jalan, 2004; Iggidr \& Bensoubaya, 1996]. Clearly $V_{k} \geq 0$ and the equality holds only when the nodes $x$ and $y$ are exactly synchronized. For the asymptotic global stability of the synchronized state, Lyapunov function should satisfy the following condition in the region of stability, $V_{k+1}<V_{k}$. This condition can also be written as,

$$
\frac{V_{k+1}}{V_{k}}<1 \text {. }
$$

Now consider version 1 of the coupling, which is given by $\left(x_{k+1}, y_{k+1}\right)=F\left(x_{k}, y_{k}\right)$, with $F$ defined by (2). Following (28), we have

$V_{k+1}=\left[f\left(x_{k}\right)-f\left(y_{k}\right)+\delta\left(y_{k}-x_{k}\right)-\varepsilon\left(x_{k}-y_{k}\right)\right]^{2}$.

If $x_{k}$ and $y_{k}$ are in the same region $R_{11}$ or $R_{33}$, then

$$
f\left(x_{k}\right)-f\left(y_{k}\right)=3\left(x_{k}-y_{k}\right)
$$

so,

$$
\begin{aligned}
V_{k+1} & =\left[3\left(x_{k}-y_{k}\right)+\left(x_{k}-y_{k}\right)(-\varepsilon-\delta)\right]^{2} \\
& =\left(x_{k}-y_{k}\right)^{2}(3-\varepsilon-\delta)^{2} .
\end{aligned}
$$

Consequently, condition (29) is equivalent to

$$
\begin{aligned}
(3-\varepsilon-\delta)^{2}<1 & \Leftrightarrow-1<3-\varepsilon-\delta<1 \\
& \Leftrightarrow 2<\varepsilon+\delta<4 .
\end{aligned}
$$

If $x_{k}$ and $y_{k}$ are in region $R_{22}$, then

$$
f\left(x_{k}\right)-f\left(y_{k}\right)=-3\left(x_{k}-y_{k}\right),
$$

so,

$$
\begin{aligned}
V_{k+1} & =\left(x_{k}-y_{k}\right)^{2}(-3-\varepsilon-\delta)^{2} \\
& =V_{k}(-3-\varepsilon-\delta)^{2} .
\end{aligned}
$$

Then condition (29) is equivalent to

$$
\begin{aligned}
(-3-\varepsilon-\delta)^{2}<1 & \Leftrightarrow-1<3+\varepsilon+\delta<1 \\
& \Leftrightarrow 2<-\varepsilon-\delta<4 .
\end{aligned}
$$


This condition is not of interest as the parameter values $\varepsilon$ and $\delta$ are positive.

Consider version 2 of the coupling given by $\left(x_{k+1}, y_{k+1}\right)=G\left(x_{k}, y_{k}\right)$, with $G$ defined by $(3)$. For this version of the coupling, the Lyapunov function is

$$
V_{k+1}=\left\{f\left[x_{k}+\delta\left(y_{k}-x_{k}\right)\right]-f\left[y_{k}+\varepsilon\left(x_{k}-y_{k}\right)\right]\right\}^{2} .
$$

If $x_{k}+\delta\left(y_{k}-x_{k}\right)$ and $y_{k}+\varepsilon\left(x_{k}-y_{k}\right)$ are in the same $R_{i i}(i=1,2,3)$ region and given that $f^{\prime \prime}=$ $f^{\prime \prime \prime}=\cdots=0$, we have by the Taylor formula at the point $c=y_{k}+\varepsilon\left(x_{k}-y_{k}\right)$ that

$$
\begin{aligned}
V_{k+1} & =\left[x_{k}+\delta\left(y_{k}-x_{k}\right)-y_{k}-\varepsilon\left(x_{k}-y_{k}\right)\right]^{2}\left[f^{\prime}(c)\right]^{2} \\
& =\left[\left(x_{k}-y_{k}\right)+\left(x_{k}-y_{k}\right)(-\varepsilon-\delta)\right]^{2}\left[f^{\prime}(c)\right]^{2} \\
& =\left(x_{k}-y_{k}\right)^{2}(1-\varepsilon-\delta)^{2}\left[f^{\prime}(c)\right]^{2} \\
& =V_{k}(1-\varepsilon-\delta)^{2}\left[f^{\prime}(c)\right]^{2} .
\end{aligned}
$$

Therefore, condition (29) is equivalent to

$$
\begin{aligned}
(1-\varepsilon-\delta)^{2}\left[f^{\prime}(c)\right]^{2}<1 & \Leftrightarrow-\frac{1}{3}<1-\varepsilon-\delta<\frac{1}{3} \\
& \Leftrightarrow 2<\alpha+\beta<4 .
\end{aligned}
$$

This means that for all initial conditions on the regions $R_{i i}(i=1,2,3)$ there is almost global synchronization, if $2<\alpha+\beta<4,(\alpha, \beta) \in[0,3]^{2}$.

In particular, this confirms our previous results, but with Lyapunov functions, we have proved the synchronization for initial conditions at any point of region $R_{i i}(i=1,2,3)$ in the case of version 2 coupling, while with the method of Lyapunov exponents, we only proved synchronization for initial conditions in a neighborhood of $S$.

\subsection{Local behavior through eigenvalues and eigenvectors}

Here, we present a study of the eigenvalues and eigenvectors of the two systems to understand the local behavior in neighborhoods of the diagonal with points in regions $R_{i j}$, whatever be $i, j$ in $1,2,3$. We only consider the parameter values corresponding to the necessary condition of synchronization. Indeed, we would like to obtain more information about the basin of synchronized states. Let us recall that the basin $B$ of an attractor $S$ at finite distance is the set of initial conditions that converge towards $S$ when the number of iterations by the considered map $T$ tends towards infinity. The basin $B$ is invariant under backward iteration of the map but not necessarily invariant by the map itself. We have the following properties: $T^{-1}(B)=B$ and $T(B) \subset B$.

\subsubsection{Version 1 coupling}

Considering the coupling map $F$ defined in (4), the Jacobian matrix of the system, in each open region $R_{i j}$ is

$$
D F\left[\begin{array}{l}
x \\
y
\end{array}\right]=\left[\begin{array}{cc}
c(x)-\delta & \delta \\
\varepsilon & c(y)-\varepsilon
\end{array}\right]
$$

where $c(x)$ and $c(y)$ are defined by (13).

First, let us consider the regions $R_{i i}, i=1,2,3$. $R_{i i}$ contains segments of the diagonal and we can look at the eigenvalues at each point $(x, x)$ of these segments. Let us recall that the eigenvalues of the Jacobian $D(x, x)$ are given by (14) and the corresponding eigenvectors by (15). In $R_{i i}, i=1,2,3$, it is easy to check that $\mu_{1}(x)$ is the eigenvalue associated to the direction on the diagonal and $\mu_{2}(x)$ to the transversal direction. When $i=1$ or $i=3$, we have $c(x)=c(y)=3$. For the parameter values $(\delta, \varepsilon)$ corresponding to the necessary condition of synchronization, $\left|\mu_{2}(x)\right|<1$, so there exists a neighborhood of the diagonal in $R_{11}$ or $R_{33}$, such that each initial condition gives rise to an iterated sequence converging toward the diagonal. Inversely, when $i=2$, we obtain $c(x)=c(y)=-3$. For the parameter values $(\delta, \varepsilon)$ corresponding to the necessary condition of synchronization, $\left|\mu_{2}(x)\right|>1$, so there exists a neighborhood of the diagonal in $R_{22}$, such that each initial condition gives rise to divergent iterated sequences. Anyhow, it is possible that those divergent iterated sequences go to another region $R_{i j}$ and reach the diagonal by another way.

Now, we consider the regions $R_{i j}, i \neq j$, from where we can approach the diagonal points $(1 / 3,1 / 3)$ and $(2 / 3,2 / 3)$. These regions are $R_{12}$, $R_{21}, R_{23}$ and $R_{32}$. In these regions we have $c(x)=$ $-c(y)$ and, taking $c=c(x)$, the Jacobian matrix becomes

$$
D F\left[\begin{array}{l}
x \\
y
\end{array}\right]=\left[\begin{array}{cc}
c-\delta & \delta \\
\varepsilon & -c-\varepsilon
\end{array}\right] .
$$

In this study, we do not consider the regions $R_{13}$ and $R_{31}$, which do not have points close to the diagonal, even if some of them can be initial conditions giving rise to trajectories converging to the diagonal. 
Let us remark that we cannot define the eigenvalues and eigenvectors exactly at the point $(1 / 3,1 / 3)$, because the map $F$ is not differentiable at this point, so we cannot define the Jacobian. Nevertheless, we can consider a directional limit and see what occurs when we are at a point $(x, y)$ in a neighborhood of the point $(1 / 3,1 / 3)$.

If we consider points $(x, y)$ in the regions $R_{i j}$ with $(i, j) \in\{(1,2),(2,1),(2,3),(3,2)\}$, we obtain the following eigenvalues $\mu_{i}$ and eigenvectors $v_{i}$

$$
\begin{aligned}
\mu_{1}= & \frac{1}{2}\left(-\delta-\varepsilon-\sqrt{(\varepsilon+\delta)^{2}+4 c^{2}+4 c \varepsilon-4 c \delta}\right), \\
\mu_{2}= & \frac{1}{2}\left(-\delta-\varepsilon+\sqrt{(\varepsilon+\delta)^{2}+4 c^{2}+4 c \varepsilon-4 c \delta}\right), \\
v_{1}= & (2 c+\varepsilon-\delta \\
& \left.-\sqrt{(\varepsilon+\delta)^{2}+4 c^{2}+4 c \varepsilon-4 c \delta}, 2 \varepsilon\right), \\
v_{2}= & (2 c+\varepsilon-\delta \\
& \left.+\sqrt{(\varepsilon+\delta)^{2}+4 c^{2}+4 c \varepsilon-4 c \delta}, 2 \varepsilon\right) .
\end{aligned}
$$

In this case, the eigenvectors depend on $(x, y)$. We only have two different situations:

(1) $R_{12}$ and $R_{32}$, where $c=3$;

(2) $R_{21}$ and $R_{23}$, where $c=-3$.

We study the eigenvalues $\mu_{i}$ with the parameters $\varepsilon$ and $\delta$ for the case where $c=3$.

In the region where we have found synchronization, $\left|\mu_{1}\right|>1$ and $\left|\mu_{2}\right|$ can be less than 1 or greater than 1 . The eigenvectors $v_{1}$ (in blue) and $v_{2}$ (in green) (see Fig. 7) have characteristic slopes depending on the region of the parameter space. So, if we take the initial point of the eigenvectors close to the point $(1 / 3,1 / 3)$, we observe that if $\varepsilon>0, v_{2}$ always points toward the direction of $R_{11}$ or $R_{22}$. If $\delta>0, v_{1}$ always points toward the direction of $R_{12}$ or $R_{21}$. We can see this situation in Fig. 7 for $\varepsilon>0$ and $\delta>0$.

We have the same kind of behavior if we consider the case $c=-3$.

To conclude this subsection, it is worth noting that if we choose initial conditions in regions $R_{i j}$ close to the diagonal, we can have trajectories that move away from the diagonal staying inside the considered region $R_{i j}$ and some others that go outside $R_{i j}$ toward another region $R_{i i}, i=1,2,3$. Moreover, in $R_{11}$ and $R_{33}$, the iterated sequences initiated close to the diagonal converge towards the diagonal, but in $R_{22}$, all iterated sequences issued

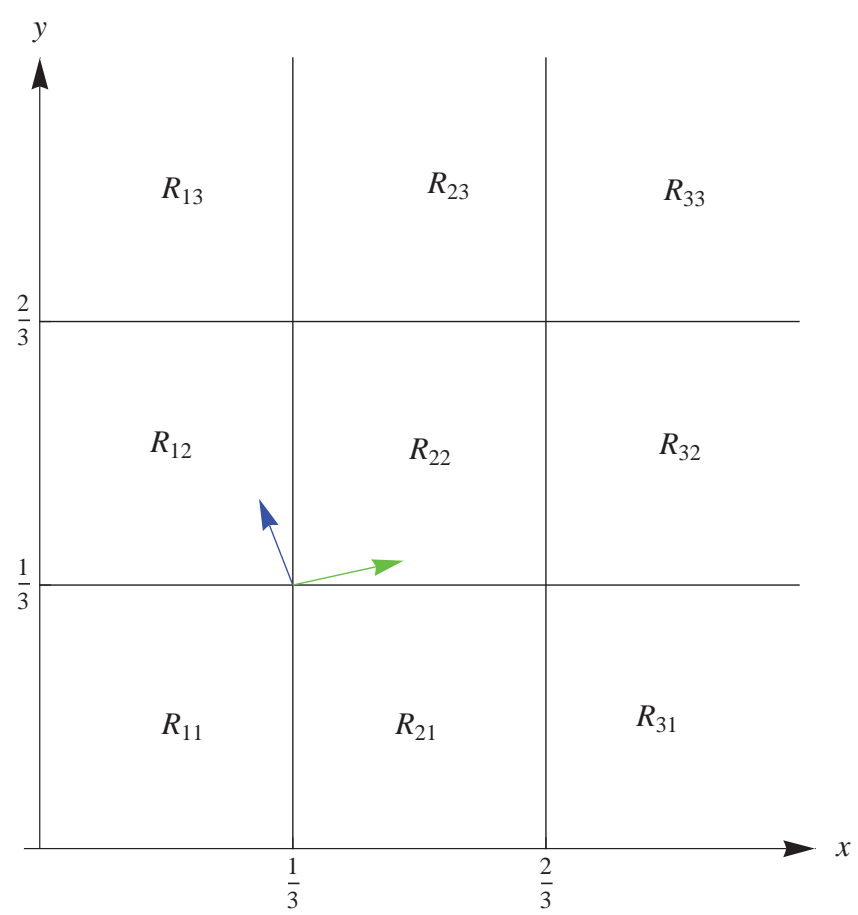

Fig. 7. For the map $F$, characteristic position of the eigenvectors, $v_{1}$ (in blue) and $v_{2}$ (in green), in the $(x, y)$-space with initial point at $(1 / 3,1 / 3)$, in regions of the parameters where $\varepsilon>0$ and $\delta>0$.

from an initial condition close to the diagonal go far away from the diagonal. In the case of the version 1 coupling we can conjecture that the basin does not contain a whole neighborhood of the diagonal.

\subsubsection{Version 2 coupling}

In this section, we consider the second coupling map $G$ defined in (5) and we intend to do the same kind of study as that for the previous section for the map $F(4)$. The Jacobian matrix of the system in each open region $R_{i j}$ is now

$$
D G\left[\begin{array}{l}
x \\
y
\end{array}\right]=\left[\begin{array}{cc}
f^{\prime}(r)(1-\delta) & f^{\prime}(r) \delta \\
f^{\prime}(s) \varepsilon & f^{\prime}(s)(1-\varepsilon)
\end{array}\right],
$$

where $r=x+\delta(y-x), s=y+\varepsilon(x-y)$ and $f$ is defined by (1).

In the regions $R_{i i}, i=1,2,3$, the eigenvalues of the Jacobian $D G$ are given by (19) and the corresponding eigenvectors by (20). As for the map (4), $\mu_{1}(x)$ is the eigenvalue associated to the direction on the diagonal and $\mu_{2}(x)$ to the transversal direction. In $R_{i i}, i=1,2,3$, it is easy to check that for the parameter values $(\delta, \varepsilon)$ (or equivalent $(\alpha, \beta))$ corresponding to the necessary condition 
of synchronization, $\left|\mu_{2}(x)\right|<1$. So there exists a neighborhood of the diagonal in $R_{11}, R_{22}$ or $R_{33}$, such that each initial condition will give rise to an iterated sequence converging toward the diagonal. This is true whatever be the value of $i=1,2,3$, oppositely to the case of the map $F(4)$.

Now, let us consider the regions $R_{i j}, i \neq j$. As in the case of the map (4), we cannot define the eigenvalues and eigenvectors exactly at the point $(1 / 3,1 / 3)$, because the map $G$ is not differentiable at this point, so we cannot define the Jacobian. Nevertheless, we can consider a directional limit and see what occurs when we are at a point $(x, y)$ very close to the point $(1 / 3,1 / 3)$ or $(2 / 3,2 / 3)$. The regions from where we can approach the diagonal points $(1 / 3,1 / 3)$ and $(2 / 3,2 / 3)$ are $R_{12}, R_{21}, R_{23}$ and $R_{32}$. In these regions, $R_{i j}$, the Jacobian matrix becomes

$$
\begin{aligned}
D G\left[\begin{array}{l}
x \\
y
\end{array}\right] & =\left[\begin{array}{cc}
c-c \delta & c \delta \\
-c \varepsilon & -c+c \varepsilon
\end{array}\right] \\
& =\left[\begin{array}{cc}
(-1)^{i+1} \alpha & (-1)^{i+1}(3-\alpha) \\
(-1)^{j+1}(3-\beta) & (-1)^{j+1} \beta
\end{array}\right],
\end{aligned}
$$

with $c=(-1)^{i+1} 3, j=i \pm 1, i, j \in 1,2,3$.

If we consider points $(x, y)$ in the regions $R_{i j}$ with $(i, j) \in\{(1,2),(2,1),(2,3),(3,2)\}$, we obtain the following eigenvalues $\mu_{i}$ and eigenvectors $v_{i}$ :

$$
\begin{aligned}
& \mu_{1}=\frac{c}{2}\left(-\delta+\varepsilon-\sqrt{(\delta-\varepsilon)^{2}+4(1-\delta-\varepsilon)}\right), \\
& \mu_{2}=\frac{c}{2}\left(-\delta+\varepsilon+\sqrt{(\delta-\varepsilon)^{2}+4(1-\delta-\varepsilon)}\right), \\
& v_{1}=\left(2-\delta-\varepsilon-\sqrt{(\delta-\varepsilon)^{2}+4(1-\delta-\varepsilon)},-2 \varepsilon\right), \\
& v_{2}=\left(2-\delta-\varepsilon+\sqrt{(\delta-\varepsilon)^{2}+4(1-\delta-\varepsilon)},-2 \varepsilon\right) .
\end{aligned}
$$

In this case, the eigenvectors do not depend on $(x, y)$, if we are in a $R_{i j}$ region with $i \neq j$.

We made a study on the eigenvalues $\mu_{i}$ with the parameters $\alpha$ and $\beta$ for the case $R_{12}$, where $c=3$ (see Fig. 8).

As in the case of version 1 coupling, the eigenvectors $v_{1}$ (in blue) and $v_{2}$ (in green) have characteristic slopes which do not depend on the region of the parameter space. So, if we put the initial point of the eigenvectors close to the point $(1 / 3,1 / 3)$, we observe that $v_{2}$ always points toward the direction of $R_{11}$ and $v_{1}$ always points toward the direction of $R_{22}$. We can see this situation in Fig. 9.

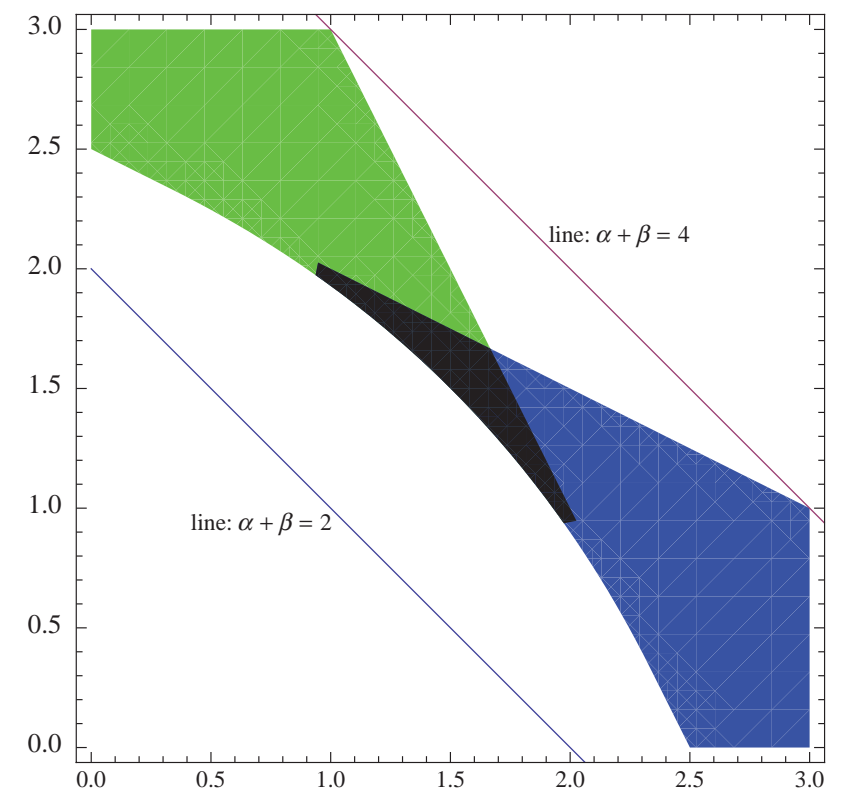

Fig. 8. For the map $G$, we can see the regions of the $(\alpha, \beta)$ plane where the eigenvalues are real and $\left|\mu_{1}\right|<1$ (in blue) and $\left|\mu_{2}\right|<1$ (in green). The black area indicates the region where both conditions are fulfilled.

If the eigenvectors are pointed towards the inside of the problematic regions $R_{i j}, i \neq j$, there would be paths that deviate from the diagonal, and this situation, for the considered parameter values, does not occur.

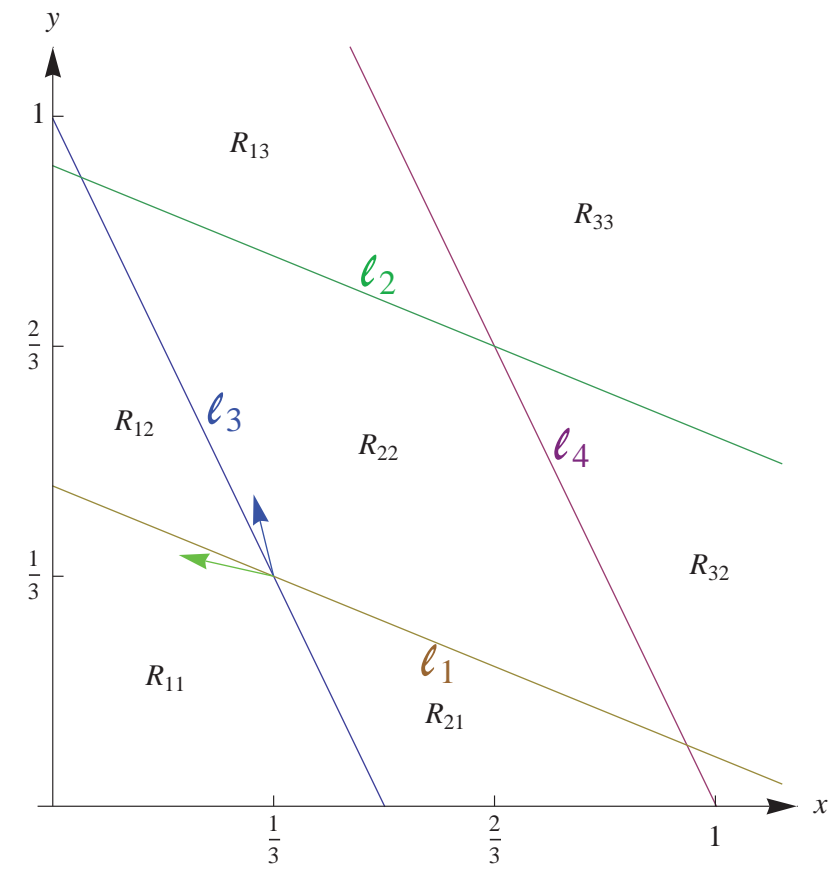

Fig. 9. Concerning the map $G$, characteristic position of the eigenvectors, $v_{1}$ (in blue) and $v_{2}$ (in green), in the $(x, y)$-space with initial point at $(1 / 3,1 / 3)$. 
So, we can conjecture that when we choose an initial condition inside $R_{i j}$, with $i \neq j$, we never move away from the diagonal, but we move away from the region $R_{i j}$ toward a region $R_{i i}$. Oppositely to the case of the map (4), in each region $R_{i i}$, the diagonal is attractive for a neighborhood located inside $R_{i i}$. So, it permits to conjecture that for the map (5), the basin of the synchronized states contains a whole neighborhood of the diagonal (except maybe sets of zero measure).

To conclude this section on the local behavior through eigenvalues and eigenvectors, by considering the eigenvalues and eigenvectors at points of the diagonal for both maps (4) and (5), we do not obtain the same result in both cases. In the case of (4) (coupling version 1), it does not seem possible to obtain a necessary and sufficient condition on parameter values for complete synchronization. Indeed, we can conjecture that the basin of the synchronized states does not contain a whole neighborhood of the diagonal. On contrary, in the case of (5) (version 2 coupling), we can conjecture it. Moreover, the basin of synchronized states should contain a whole neighborhood of the diagonal.

\section{Basins}

In this paragraph, we wish to present some properties of the basins of synchronized states for the maps $F(4)$ and $G(5)$. The condition giving possible values of parameters to obtain complete synchronization is a necessary condition, as previously explained. In previous studies, it is also said that all initial conditions taken in the phase space do not necessarily give rise to synchronization (that means trajectories converging towards diagonal). Indeed, sometimes, it can be necessary to remove a dense set of zero measure to obtain the basin of the synchronized states. Anyhow, we are going to see that, depending upon the type of maps, the basins can have very different shapes and are not necessarily constituted by the whole phase space. Sometimes, it is not only required to remove a set of zero measure, but larger sets. In both cases, the basins can be riddled. We consider the two cases of versions 1 and 2, built from the bimodal map and we consider the basin of synchronized states. Our basins have been obtained by numerical simulations. Such simulations, coupled with geometrical properties of critical lines, as introduced in [Mira et al., 1996], permit to explain the shapes of basins.

\subsection{Basins of the map $F$}

We consider the map $F$. Let us first remark that we can extend the definition of $F:[0,1]^{2} \rightarrow[0,1]^{2}$ to $F: R^{2} \rightarrow R^{2}$ (using $f(x)=|1-| 3 x-1||$, which can be defined on $R$ ). It will permit to have a better understanding of the evolution of the basin of attraction. Previous studies on two-dimensional maps [Mira et al., 1996] have shown that the study of critical lines is of high interest to obtain information on the evolution of the basin shape. So, we consider the straight lines $l_{1}: y=1 / 3, l_{2}: x=1 / 3$, $l_{3}: y=2 / 3, l_{4}: x=2 / 3$ and their images by $F$, that are the critical lines of $F$. We can define four points: $A, B, C, D$, which are intersection points between the lines $l_{i}, i=1,2,3,4$ (see Figs. 10-12). $A=l_{1} \cap l_{2}, B=l_{3} \cap l_{4}, C=l_{2} \cap l_{3}, D=l_{1} \cap l_{4}$. The coordinates of these points are $A(1 / 3,1 / 3)$, $B(2 / 3,2 / 3), C(1 / 3,2 / 3)$ and $D(2 / 3,1 / 3)$. We can define $L_{2}=F\left(l_{2}\right)$, which we are interested in and whose equation is

$$
\left\{\begin{array}{l}
\text { if } \delta=0: x=1, y \geq-\frac{\varepsilon}{3} \\
\text { if } \delta \neq 0: y=|1-| \frac{3(x-1)}{\delta}||-\frac{\varepsilon(1-x)}{\delta} .
\end{array}\right.
$$

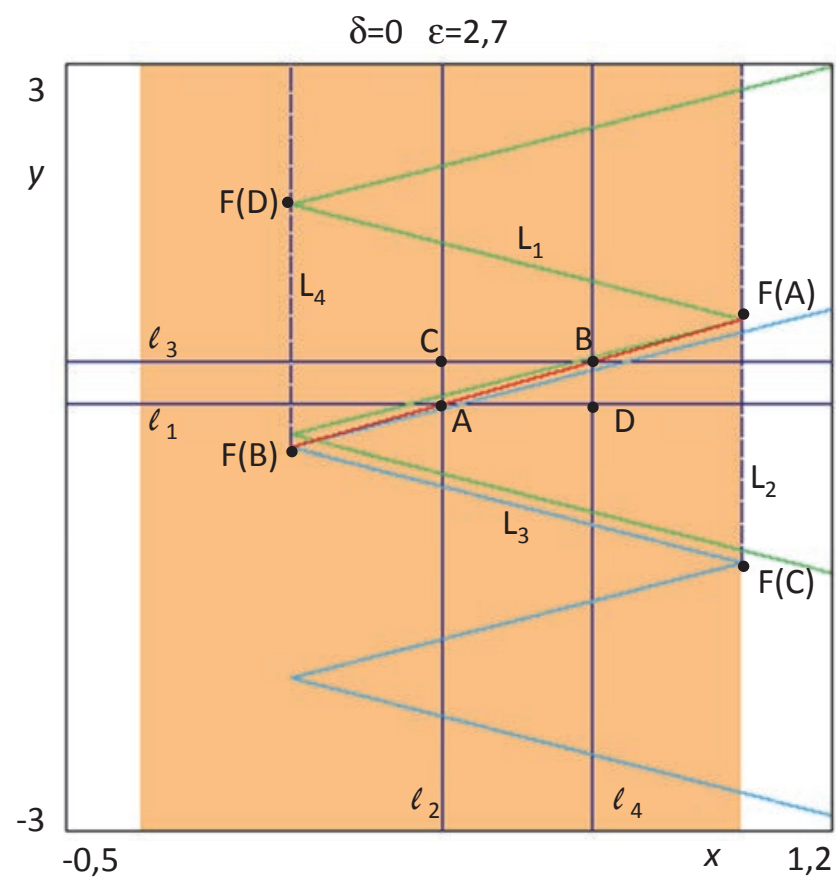

Fig. 10. In the state space $(x, y), \delta=0, \varepsilon=2.7$, in beige, the basin of the chaotic attractor on the diagonal (in red) for the map $F$. The critical line $L_{1}$ is in green and $L_{3}$ in light blue. $L_{2}$ and $L_{4}$ are dashed lines. States are synchronized, the synchronization is global. 


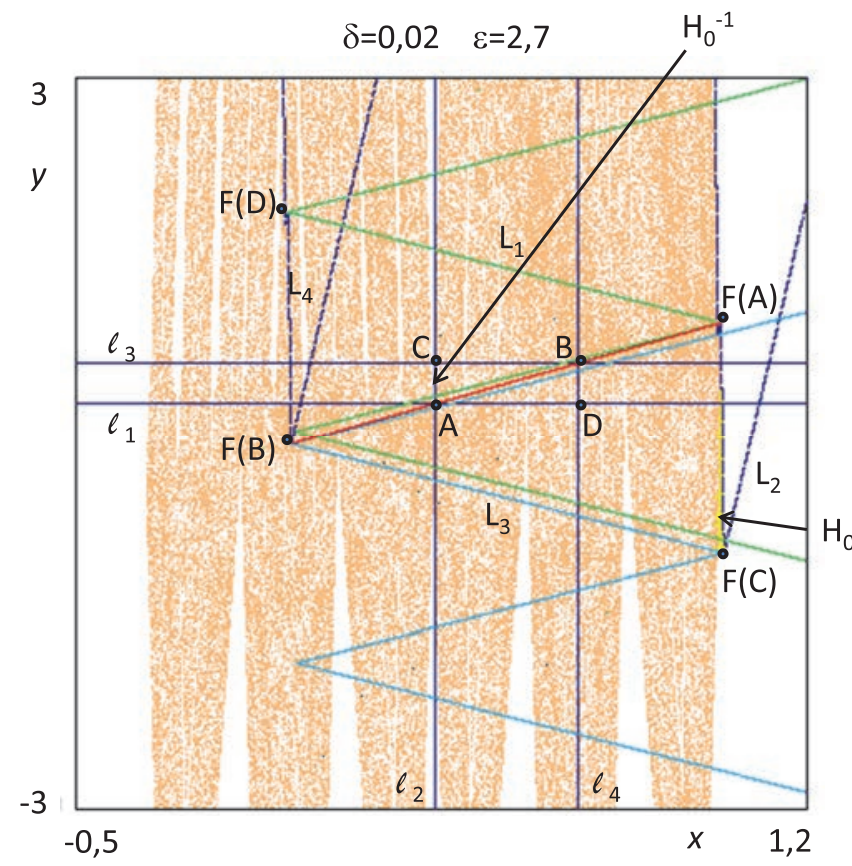

Fig. 11. In the state space $(x, y), \delta=0.02, \varepsilon=2.7$, in beige, the basin of the chaotic attractor on the diagonal (in red) for the map $F$. The hole $H_{0}$ is in yellow, its preimage $H_{0}^{-1}$ is located close to the point $C$ and the lines $l_{2}$ and $l_{3}$. States are synchronized, but the synchronization is not global, due to the holes inside the basin.

This curve is made up of four different pieces when $\delta \neq 0$ :

$$
y= \begin{cases}-1+\frac{(3-\varepsilon)(x-1)}{\delta}, & \text { if } 1+\frac{\delta}{3} \leq x \\ 1+\frac{(3+\varepsilon)(1-x)}{\delta}, & \text { if } 1 \leq x<1+\frac{\delta}{3} \\ 1+\frac{(3-\varepsilon)(x-1)}{\delta}, & \text { if } 1-\frac{\delta}{3} \leq x<1 \\ -1+\frac{(3+\varepsilon)(1-x)}{\delta}, & \text { if } x<1-\frac{\delta}{3} .\end{cases}
$$

We can define the curves $L_{1}, L_{3}$ and $L_{4}$ in the same way. The point $F(C)$ belongs to $L_{2} \cap L_{3}$ and its coordinates are $(1+\delta / 3,-\varepsilon / 3)$. When $\delta=0$, $F(C)$ is the point $(1,-\varepsilon / 3)$ and is located on the boundary of the basin (see Fig. 10). When $\delta$ becomes slightly positive $(\delta=0.02$ on Figs. $11-13)$, the point $F(C)$ is located outside the basin. A domain $H_{0}$ is created (Fig. 12), the preimages of any rank of which are sets of initial conditions of divergent iterated sequences located inside the beige basin. As $H_{0}$ is limited by $F(C)$ and pieces of the

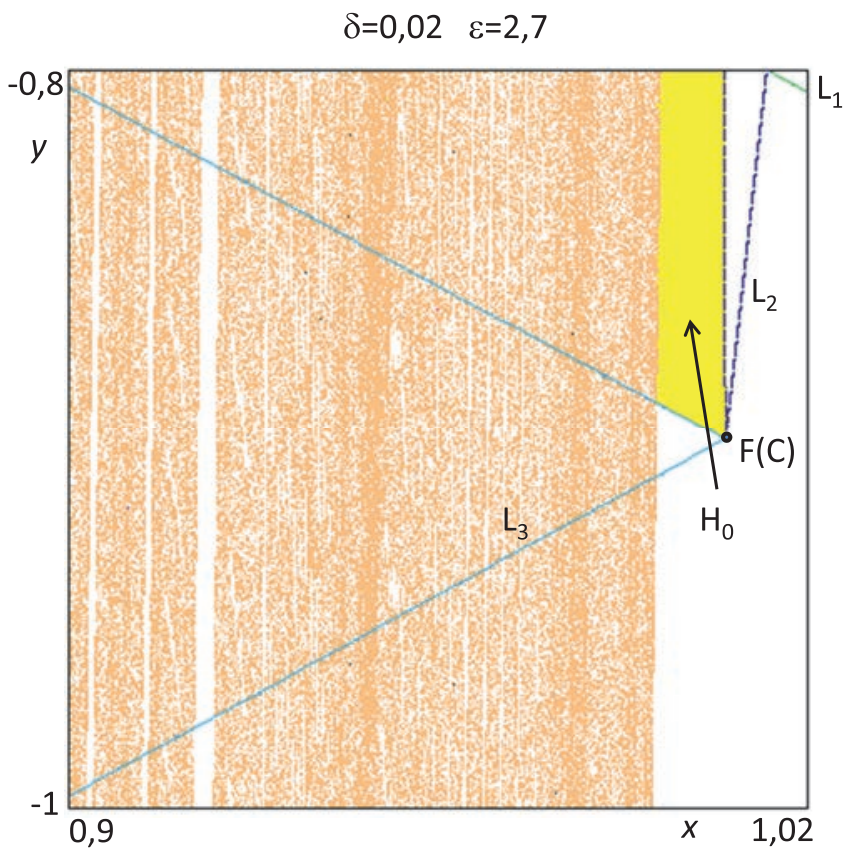

Fig. 12. Enlargment of Fig. 11. One can see the region $H_{0}$ in yellow, which is limited by pieces of $L_{2}$ and $L_{3}$, the point $F(C)$ and the boundary of the basin.

curves $L_{2}$ and $L_{3}$, the rank-one preimage of $H_{0}$, $H_{0}^{-1}$, is limited by $C$ and pieces of $l_{2}$ and $l_{3}$ and located below $C$ (situation due to the branch of $L_{2}$ which is involved in the creation of $H_{0}$ ). In this case, there can be a set of nonzero measure inside

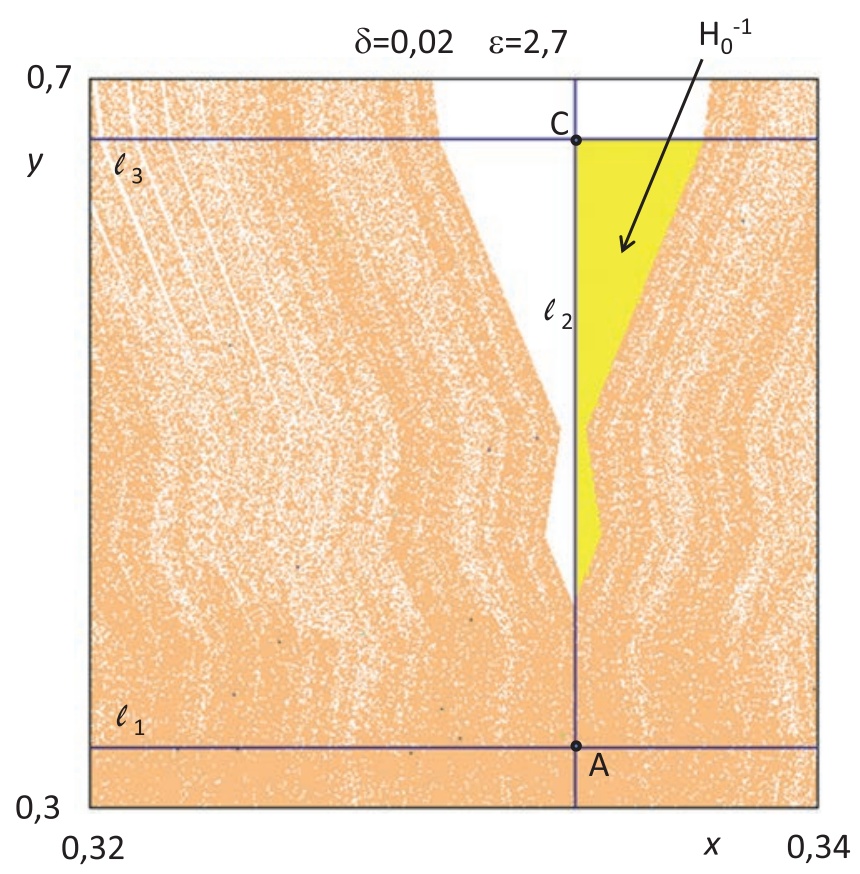

Fig. 13. Enlargment of Fig. 11. One can see the region $H_{0}^{-1}$ in yellow, which is limited by pieces of $l_{2}$ and $l_{3}$, the point $C$ and the boundary of the basin. 


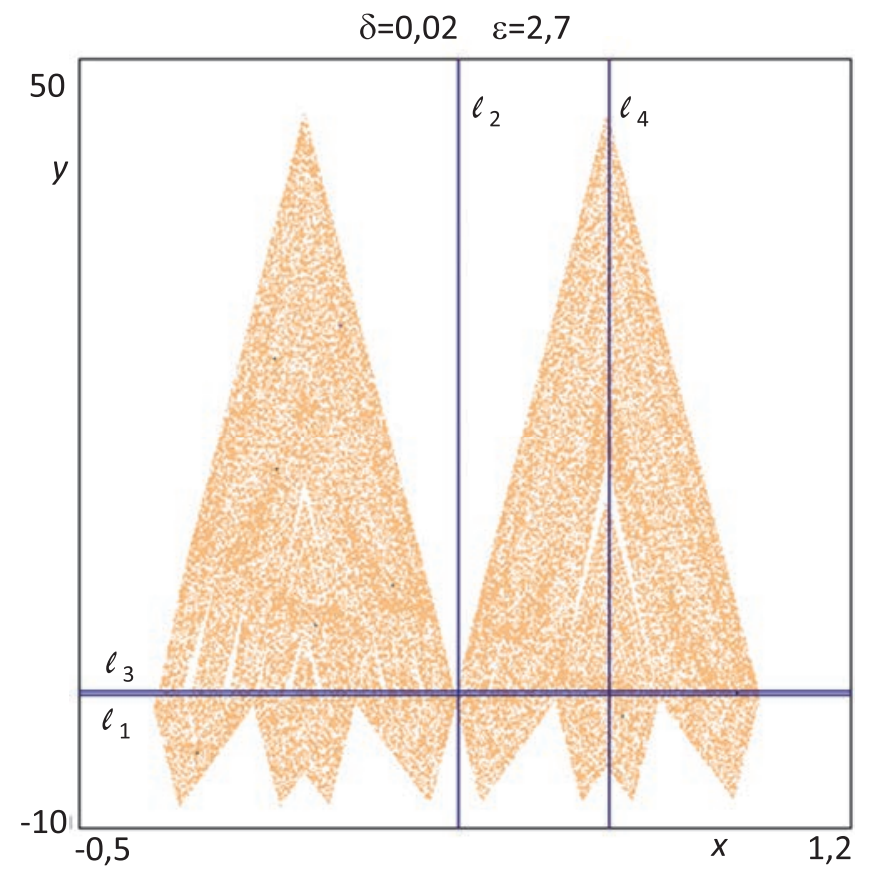

Fig. 14. In the state space $(x, y), \delta=0.02, \varepsilon=2.7$, in beige, the total basin in $\mathbb{R}^{2}$ of the chaotic attractor on the diagonal for the map $F$.

the basin, which is a set of initial conditions giving divergent trajectories. So, synchronization occurs, but is not global. The basin of the synchronized states is fractal and contains holes or tongues of

\section{$\delta=2,4 \quad \varepsilon=0,6$}

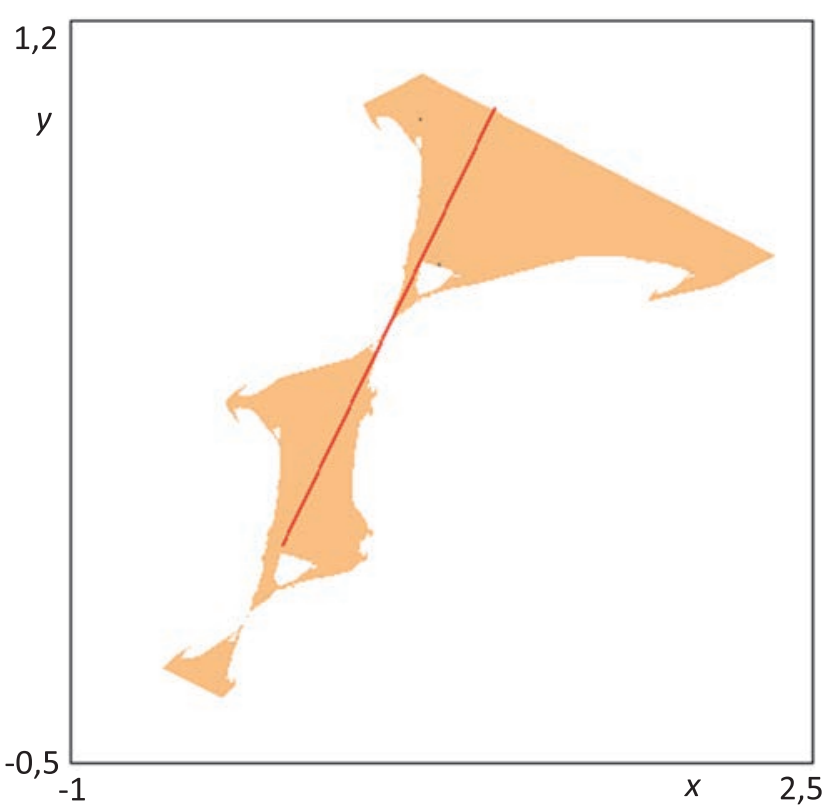

Fig. 15. In the state space $(x, y), \delta=2.4, \varepsilon=0.6$, in beige, the multiply connected basin in $\mathbb{R}^{2}$ of the chaotic attractor on the diagonal (in red) for the map $F$. $\delta=2,5 \quad \varepsilon=0,6$

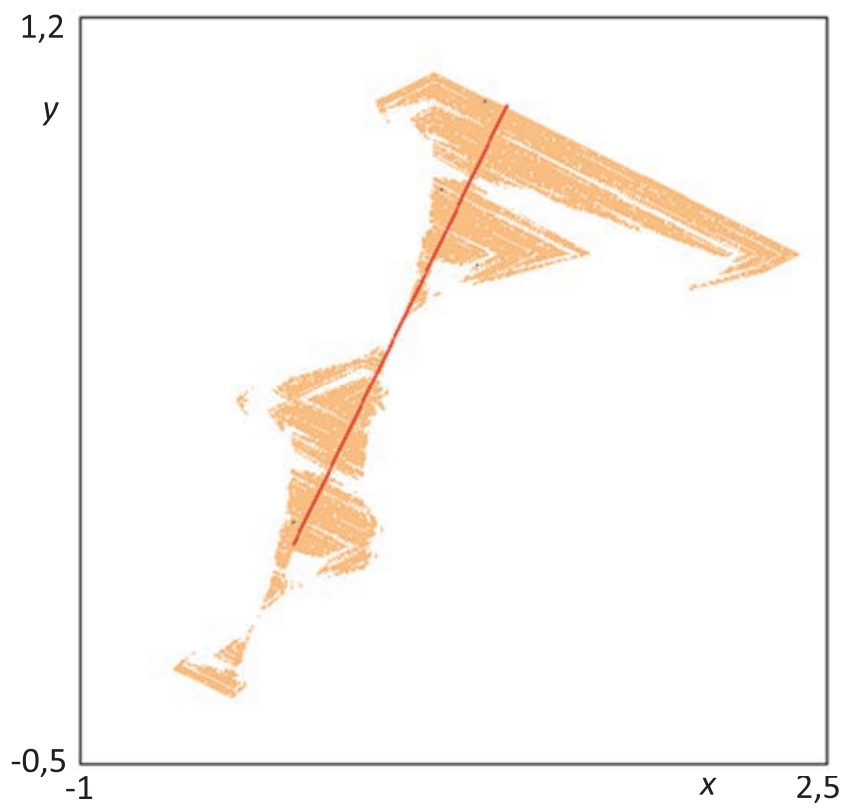

Fig. 16. In the state space $(x, y), \delta=2.5, \varepsilon=0.6$, in beige, the basin in $\mathbb{R}^{2}$ of the chaotic attractor on the diagonal (in red) for the map $F$. The basin has a fractal boundary.

initial conditions corresponding to divergent iterated sequences said to be multiply connected [Mira et al., 1996] (Figs. 11, 12 and 14). Figures 15 and 16 give examples of other shapes of the basin of synchronized states.

\subsection{Basins of the map $G$}

In the case of version 2, we do not observe the same phenomena for the basins than in the case of version 1 . Indeed, we only consider parameter values for which the square $[0,1]^{2}$ is mapped into itself, so, initial conditions do not give rise to divergent iterated sequences. All trajectories stay inside $[0,1]^{2}$. Main initial conditions converge to the diagonal and we have complete synchronization, except, as it is said in [Hasler \& Maistrenko, 1987], for a set of initial conditions of zero measure.

\section{Conclusion}

Following the works by Hasler and Maistrenko [1987], we have considered two kinds of couplings (1 and 2) between two identical one-dimensional bimodal maps, in order to study the synchronization. So, we have studied two two-dimensional piecewise linear maps, defined on $\mathbb{R}^{2}$ (map $F$ ) or $[0,1]^{2}(\operatorname{map} G)$ and depending upon two parameters. The complete synchronization corresponds to 
the existence of an attractor on the diagonal. We have tried different tools in order to obtain conditions on parameter values for having synchronized states (natural transversal Lyapunov exponent, Lyapunov functions, eigenvalues and eigenvectors, numerical simulations). Then, we have obtained different results for both maps. A necessary and sufficient condition of stability and global results have been given for the map $G$ (coupling 2), whereas it was not possible for the map $F$ (coupling 1 ). Complete synchronization can be proven for $F$ but only in a neighborhood of the diagonal. It has been only possible to give a global condition in two regions $R_{11}$ and $R_{33}$. The numerical simulations, coherent with the theoretical results, have indicated that the basins are not necessarily a whole domain of initial conditions in the case of coupling 1 , they can be fractal and we can obtain sets of nonzero measure for the basins of convergent iterated sequences (synchronized states) and divergent iterated sequences, whereas, for version 2 coupling, the basin of synchronized states is constituted by the whole square $[0,1]^{2}$, except at most a set of zero measures. This study could be extended to other kinds of piecewise differentiable two-dimensional maps. Indeed, many models using two-dimensional maps permit to obtain synchronization, the questions which arise are then how can we define the regions of synchronization in parameter spaces? How can we obtain conditions on parameters and states? How can we have information on basins? Can we obtain complete and/or almost global synchronization? Some tools used in this paper could be useful for such other studies.

\section{Acknowledgments}

D. Fournier-Prunaret would like to thank INSA Toulouse, France and University of Evora, Portugal for having supported this work.

\section{References}

Alatriste, F. \& Mateos, J. L. [2006] "Phase synchronization in tilted deterministic ratchets," Physica A $\mathbf{3 7 2}$, $263-271$.

Alexander, J. C., Yorke, J. A. \& You, Z. [1992] "Riddled basins," Int. J. Bifurcation and Chaos 2, 795-813.

Ashwin, P., Buescu, J. \& Stewart, J. [1996] "From attractor to chaotic saddle: A tale of transverse instability," Nonlinearity 9, 703-737.
Boccaletti, S., Kurths, J., Osipov, G., Valladares, D. L. \& Zhou, C. S. [2002] "The synchronization of chaotic systems," Phys. Rep. 366, 1-101.

Canale, E. \& Monzón, P. [2008] "Almost global synchronization of symmetric Kuramoto coupled oscillators," Systems Structure and Control, pp. 167-190.

Caneco, A., Rocha, J. L. \& Gracio, C. [2009] "Topological entropy in the synchronization of piecewise linear and monotone coupled maps. Coupled Duffing oscillators," Int. J. Bifurcation and Chaos 19, 3855-3868.

Caneco, A., Rocha, J. L., Gracio, C. \& Fernandes, S. [2011] "Some conjectures about the synchronizability and the topology of networks," Frontiers in the Study of Chaotic Dynamical Systems with Open Problems, Vol. 1, World Scientific Series on Nonlinear Science, Series B, pp. 117-147.

Cao, J. \& Lu, J. [2006] "Adaptive synchronization of neural networks with or without time-varying delays," Chaos 16, 013133.

Hasler, M. \& Maistrenko, Y. L. [1987] "An introduction to the synchronization of chaotic systems: Coupled skew tent maps," IEEE Trans. Circuits Syst.-I: Fund. Th. Appl. 44, 856-866.

Iggidr, A. \& Bensoubaya, M. [1996] "Stability of discrete time systems," Research Report Institut National de Recherche en Informatique et en Automatique (INRIA), No. 3003.

Jalan, S. [2004] "Some studies of growing networks with non-linear dynamical elements," PhD thesis, Mohanlal Sukhadia University, Udaipur, India.

Li, X. \& Chen, G. [2003] "Synchronization and desynchronization of complex dynamical networks: An engineering viewpoint," IEEE Trans. Circuits Syst. 50, 1381-1390.

Luo, A. \& Min, F. [2011] "The mechanism of a controlled pendulum synchronizing with periodic motions in a periodically forced, damped Duffing oscillator," Int. J. Bifurcation and Chaos 21, 1813-1829.

Manjunath, G. \& Fournier-Prunaret, D. [2011] "Strong persistence of an attractor and generalized partial synchronization in a coupled chaotic system," Chaos 21, 023110 .

Mira, C., Gardini, L., Barugola, A. \& Cathala, J. C. [1996] Chaotic Dynamics in Two-Dimensional Non Invertible Maps, World Scientific Series on Nonlinear Science. Series A: Monographs and Treatises, Vol. 20 (World Scientific, Singapore).

Osipov, G. V., Kurths, J. \& Zhou, C. [2007] Synchronization in Oscillatory Networks (Springer, Berlin).

Pecora, L. M. \& Carroll, T. L. [1990] "Synchronization in chaotic systems," Phys. Rev. Lett. 64, 821-824.

Pecora, L. M. \& Carroll, T. L. [1991] "Driving systems with chaotic signals," Phys. Rev. A 44, 2374-2383. 
Pikovsky, A., Rosenblum, M. \& Kurths, J. [2001] Synchronization: A Universal Concept in Nonlinear Sciences (Cambridge University Press).

Sherman, A., Rinzel, J. \& Keizer, J. [1988] "Emergence of organized bursting in clusters of pancreatic betacells by channel sharing," Biophys. J. 54, 411-425.

Shuai, J. W., Wong, K. W. \& Cheng, L. M. [1997] "Synchronization of spatiotemporal chaos with positive conditional Lyapunov exponents," Phys. Rev. E 56, 2272 .

Srinivasan, K., Senthilkumar, D. V., Raja Mohamed, I., Murali, K., Lakshmanan, M. \& Kurths, J. [2012] "Anticipating, complete and lag synchronizations in RC phase-shift network based coupled Chua's circuits without delay," Chaos 22, 023124.

Suresh, R., Senthilkumar, D. V., Lakshmanan, M. \& Kurths, J. [2012] "Global and partial phase synchronizations in arrays of piecewise linear timedelay systems," Int. J. Bifurcation and Chaos 22, 1250178.

Toiya, M., Gonzalez-Ochoa, H., Vanag, V., Fraden, S. \& Epstein, I. [2010] "Synchronization of chemical microoscillators," J. Phys. Chem. Lett. 1, 1241-1246.

Wieland, P., Sepulchre, R. \& Allgower, F. [2011] "An internal model principle is necessary and sufficient for linear output synchronization," Automatica 47, 10681074.

Wu, Z., Chen, G. \& Fu, X. [2012] "Synchronization of a network coupled with complex-variable chaotic systems," Chaos 22, 023127.

Yamada, T. \& Fujisaka, H. [1984] "Stability theory of synchronized motion in coupled-oscillator systems," Progr. Theoret. Phys. 72, 885-894. 